\title{
Polynomial spline spaces of non-uniform bi-degree on T-meshes: combinatorial bounds on the dimension
}

\section{Deepesh Toshniwal $^{1}$ (D) . Bernard Mourrain ${ }^{2} \cdot$ Thomas J. R. Hughes $^{3}$}

Received: 6 October 2020 / Accepted: 17 November 2020 /

Published online: 2 February 2021

(C) The Author(s) 2021

\begin{abstract}
Polynomial splines are ubiquitous in the fields of computer-aided geometric design and computational analysis. Splines on T-meshes, especially, have the potential to be incredibly versatile since local mesh adaptivity enables efficient modeling and approximation of local features. Meaningful use of such splines for modeling and approximation requires the construction of a suitable spanning set of linearly independent splines, and a theoretical understanding of the spline space dimension can be a useful tool when assessing possible approaches for building such splines. Here, we provide such a tool. Focusing on T-meshes, we study the dimension of the space of bivariate polynomial splines, and we discuss the general setting where local mesh adaptivity is combined with local polynomial degree adaptivity. The latter allows for the flexibility of choosing non-uniform bi-degrees for the splines, i.e., different bi-degrees on different faces of the T-mesh. In particular, approaching the problem using tools from homological algebra, we generalize the framework and the discourse presented by Mourrain (Math. Comput. 83(286):847-871, 2014) for uniform bi-degree splines. We derive combinatorial lower and upper bounds on the spline space dimension and subsequently outline sufficient conditions for the bounds to coincide.
\end{abstract}

Communicated by: Larry L. Schumaker

Deepesh Toshniwal

d.toshniwal@tudelft.nl

Bernard Mourrain

bernard.mourrain@inria.fr

Thomas J. R. Hughes

hughes@oden.utexas.edu

1 Delft Institute of Applied Mathematics, Delft University of Technology, Delft, The Netherlands

2 Inria Sophia Antipolis Méditerranée, Université Côte d'Azur, Valbonne, France

3 Oden Institute for Computational Engineering and Sciences, University of Texas at Austin, Austin, TX, USA 
Keywords Smooth splines $\cdot$ T-Meshes $\cdot$ Non-uniform degrees $\cdot$ Dimension formula $\cdot$ Homological algebra

Mathematics Subject Classification (2010) 14Q99 - 13P25 - 68W30 - 65D07 · $65 \mathrm{D} 17$

\section{Introduction}

Standard B-spline parameterizations of surfaces in computer-aided geometric design are defined on a grid of nodes over a rectangular domain. These representations are also the basis of isogeometric analysis which generalizes high-order finite element methods in numerical simulations [9]. However, grid structures do not allow complex shapes to be easily resolved. They also preclude the flexibility of performing local refinements for improving the error in numerical simulations. To address these issues, meshes with T-junctions-also called T-meshes - and polynomial and rational splines on such meshes have been investigated for performing both geometric modeling and isogeometric analysis. Classically, uniform degree splines, i.e., piecewise polynomial functions of uniform degree on the faces, have been studied and developed on T-meshes with the intent of using T-junctions for locally increasing the resolution offered by the spline space. An alternate strategy to improve the approximation power of splines is to increase the degree in a localized manner, providing in this way more spline basis functions and more degrees of freedom for better approximation in the desired regions. In this paper, motivated by applications for isogeometric finite element methods, we study the space of piecewise polynomials functions on a T-mesh with different bi-degrees on its faces and different regularities across its edges. In particular, we analyze the dimension of these functional spaces, thus providing a tool that can help identify when a given set of linearly independent splines spans the full space.

Computing the dimensions of multivariate spline spaces is a highly non-trivial task and involves an intimate interplay of algebra, topology, and geometry. Some of the notable early contributions to this problem focused on splines on simplicial meshes. The first studies were conducted by Strang [31, 32]; Schumaker [25] provided lower bounds on the dimension of splines on planar triangulations; Billera [2] brought homological algebra to bear on the problem; and Schenck and Stillman [24] modified the approach of [2] to devise a more tractable framework for studying the dimension problem. Those earlier papers have paved the way for a multitude of developments, and this direction of research is now a classical topic in the field of approximation theory. In particular, over the past four decades, polynomial and nonpolynomial spline spaces have been analyzed and characterized on a wide variety of meshes (e.g., polygonal, polyhedral, curvilinear). Nevertheless, in the following, we describe the problem and discuss the literature only for splines on T-meshes in the interest of an abbreviated but focused narrative.

Several works focused on the construction of spline functions and the analysis of spline function spaces on T-meshes exist, mainly motivated by applications in isogeometric analysis. So-called T-splines, which are B-spline functions defined on 
domains with a T-mesh structure, have been investigated for their flexibility of representing shapes [28], for isogeometric analysis [1] and for functional approximation [26]. However, linear dependencies of the blending functions involved in T-spline constructions have been observed [8]. To remedy this problem, a special sub-family of T-splines, called analysis suitable T-splines, has been developed, by imposing sufficient constraints on the T-mesh [7, 20,27].

The construction of so-called LR-splines defined on T-meshes and based on knot sub-grids has been proposed in [12]. Their use in isogeometric analysis has been further investigated in [17], including an analysis of the linear independency of the blending functions [6]. Another type of splines, so-called hierarchical B-splines, has been investigated in $[10,13,14,18]$. They are defined by recursive subdivisions of quadrangular faces, producing nested spaces of splines functions and providing simple schemes for performing local refinements.

In general, the dimension of the spaces of splines on T-meshes can be unstable, i.e., it can depend on the global geometry of the T-mesh [19, 22]. Since any efficient constructive approach must rely only on local data for building spline functions, this instability in the dimension necessitates identification of configurations where the spline space dimension is a priori guaranteed to be stable. In this direction, a detailed study of spline spaces on general T-meshes has been presented in [22] using homological techniques, which go back to [2]. Results from [22] were used in [11] to devise a refinement strategy for LR-splines that ensures that the entire spline space is spanned by LR B-splines at each stage of refinement. The dimension of Tchebycheffian spline spaces over planar T-meshes, which involve non-polynomial functions, has been investigated in [4,5], exploiting the same homological techniques as in [22].

In all the works referenced above, only uniform degree splines are considered on the T-meshes. Spline spaces on triangulations with non-uniform degree have been recently studied in [35] using homological techniques, and the interest of using nonuniform degree T-splines in isogeometric analysis has been shown in [21, 33].

Here, we analyze in detail splines spaces over general T-meshes when non-uniform polynomial bi-degrees are chosen on the faces, thus accounting for local degree adaptivity in conjunction with local mesh adaptivity. We provide combinatorial lower and upper bounds on the dimensions of such spline spaces and outline sufficient conditions for the bounds to coincide. These sufficient conditions are equivalent to geometric conditions that need to be satisfied by the T-meshes. The approach is based on homological techniques and generalizes the framework presented in [22] to the case of non-uniform polynomial bi-degree distributions. As part of the approach, we perform a degree-based decomposition of the mesh into nested regions and this allows us to untangle the contributions of different bi-degrees to the spline space dimension. The main results on the lower and upper bounds of the dimension of these spline spaces (Theorems 6.3, 6.4, and 7.1) involve homological invariants of the nested regions associated to the different bi-degrees. As mentioned previously, the theoretical results presented here can be used to identify when a given set of linearly independent splines spans the full spline space. Conversely, given a constructive approach that aims to produce linearly independent splines over T-meshes using only local data, computation of the associated spline space dimension can help identify cases where the splines produced by the approach cannot be linearly independent. 
This is crucial for devising constructive approaches that can be robustly employed for performing isogeometric analysis.

The layout of the paper is as follows. We start by introducing preliminary concepts and notation about T-meshes and non-uniform bi-degree spline spaces on such meshes in Section 2. Thereafter, we introduce the topological complexes that form the main object of our analysis in Section 3; in particular, Section 3.3 provides an overview of our approach to the problem at hand. Sections 4 and 5 take a closer look at the topological complexes introduced in Section 3, and the results presented therein are used in Section 6 to provide bounds on the spline space dimension (Theorems 6.3 and 6.4). Section 7 contains Theorem 7.1, which outlines sufficient conditions for the bounds derived in Section 6 to coincide. We also discuss the notion of maximal segment weights, generalized from [22]. This notion helps provide a geometric criterion that is useful when computing the spline space dimension. Finally, Section 8 provides examples of the theory developed here. We would like to mention here that computations using Macaulay2 [15] went hand-in-hand with the research presented here.

\section{Planar T-meshes and polynomials}

In the following, we define the basic concepts associated with planar T-meshes, and thereafter present some preliminary results on polynomials. We will proceed as in [22], albeit in the setting of non-uniform degree spline spaces.

\subsection{T-meshes}

Definition 2.1 (T-mesh) A T-mesh $\mathcal{T}$ of $\mathbb{R}^{2}$ is defined as:

- a finite set $\mathcal{T}_{2}$ of closed axis-aligned rectangles $\sigma$ of $\mathbb{R}^{2}$, called 2-cells or faces,

- a finite set $\mathcal{T}_{1}$ of closed axis-aligned segments $\tau$, called 1-cells or edges, included in $\cup_{\sigma \in \mathcal{T}_{2}} \partial \sigma$, and,

- a finite set, $\mathcal{T}_{0}$, of points $\gamma$, called 0 -cells or vertices, included in $\cup_{\tau \in \mathcal{T}_{1}} \partial \tau$,

such that

- $\sigma \in \mathcal{T}_{2} \Rightarrow \partial \sigma$ is a finite union of elements of $\mathcal{T}_{1}$,

- $\sigma, \sigma^{\prime} \in \mathcal{T}_{2} \Rightarrow \sigma \cap \sigma^{\prime}=\partial \sigma \cap \partial \sigma^{\prime}$ is a finite union of elements of $\mathcal{T}_{1} \cup \mathcal{T}_{0}$, and,

- $\quad \tau, \tau^{\prime} \in \mathcal{T}_{1}$ with $\tau \neq \tau^{\prime} \Rightarrow \tau \cap \tau^{\prime}=\partial \tau \cap \partial \tau^{\prime} \subset \mathcal{T}_{0}$.

The domain of the T-mesh is assumed to be connected and is defined as $\Omega:=$ $\cup_{\sigma \in \mathcal{T}_{2}} \sigma \subset \mathbb{R}^{2}$.

Sets of horizontal and vertical edges will be denoted by ${ }^{h} \mathcal{T}_{1}$ and ${ }^{v} \mathcal{T}_{1}$, respectively. Edges of the T-mesh are called interior edges if they intersect $\stackrel{\circ}{\Omega}$, and boundary edges otherwise. The set of interior edges will be denoted by $\stackrel{\circ}{\mathcal{T}}_{1}$; and the sets of interior horizontal and vertical edges will be denote by $h \stackrel{\circ}{\mathcal{T}}_{1}$ and ${ }^{v} \stackrel{\circ}{\mathcal{T}}_{1}$, respectively. Similarly, if a vertex is in $\stackrel{\Omega}{\Omega}$, it will be called an interior vertex, and a boundary vertex otherwise. 
The set of interior vertices will be denoted by $\stackrel{\circ}{\mathcal{T}}_{0}$. We will denote the number of $i$-cells with $\mathfrak{t}_{i}:=\# \mathcal{T}_{i}$; the number of interior $i$-cells with $\stackrel{\circ}{\mathfrak{t}}_{i}:=\# \stackrel{\circ}{\mathcal{T}}_{i}$.

Assumption 1 The domain $\Omega$ is simply connected, and $\stackrel{\circ}{\Omega}$ is connected.

\subsection{Splines on T-meshes}

We will now define spaces of piecewise-polynomial splines on the planar T-meshes introduced above. To do so, we will first define a map that specifies relative polynomial degrees on the faces of $\mathcal{T}$, and a second map that specifies the smoothness across its edges. Note that these maps are assumed to be known/fixed throughout this document and, when needed, we will omit mentioning them explicitly in order to simplify notation.

To define a non-uniform degree spline space on $\mathcal{T}$, we need as input a degreedeficit distribution that will later help us specify non-uniform degrees on $\mathcal{T}$. We represent the degree-deficits in the form of a map that specifies an ordered pair of non-negative integers to each face of $\mathcal{T}$,

$$
\begin{aligned}
\Delta \boldsymbol{m}: & \mathcal{T}_{2} \rightarrow \mathbb{Z}_{\geq 0}^{2}, \\
& \sigma \mapsto \Delta \boldsymbol{m}(\sigma) .
\end{aligned}
$$

It is assumed that $\mathcal{D}:=\left\{\boldsymbol{\Delta} \boldsymbol{m}(\sigma): \sigma \in \mathcal{T}_{2}\right\}$ can be totally ordered using the relation $\leq \mathcal{D}$ defined as

$$
\left(a_{1}, a_{2}\right) \leq \mathcal{D}\left(b_{1}, b_{2}\right) \Leftrightarrow a_{1} \leq b_{1} \wedge a_{2} \leq b_{2},
$$

and that $(0,0) \in \mathcal{D}$. Given a degree-deficit distribution as above, we build the following sequence $\Delta \boldsymbol{m}_{i}, i=0, \ldots, \mathfrak{l}$,

$$
\min \mathcal{D}=(0,0)=: \Delta \boldsymbol{m}_{0}<\Delta \boldsymbol{m}_{1}<\cdots<\Delta \boldsymbol{m}_{\mathfrak{l}}:=\max \mathcal{D},
$$

such that

$$
\Delta \boldsymbol{m}_{i}-\Delta \boldsymbol{m}_{i-1}=: \Delta \boldsymbol{n}_{i} \in\{(1,0),(0,1),(1,1)\} .
$$

Note that all comparisons carried out above are with respect to the ordering in Equation (2.2). We will denote the components of $\boldsymbol{\Delta} \boldsymbol{m}_{i}$ and $\boldsymbol{\Delta} \boldsymbol{n}_{i}$ with $\left(\Delta m_{i 1}, \Delta m_{i 2}\right)$ and $\left(\Delta n_{i 1}, \Delta n_{i 2}\right)$, respectively.

The map $\Delta \boldsymbol{m}$ will help specify the bi-degree of polynomials on a face $\sigma \in \mathcal{T}_{2}$. Given $\boldsymbol{m} \in \mathbb{Z}_{\geq 0}^{2}$, we define the bi-degrees $\boldsymbol{m}_{\square}, \square \in \mathcal{T}_{2} \cup \mathcal{T}_{1} \cup \mathcal{T}_{0}$, as

$$
m_{\square}:=m-\Delta m(\square),
$$

where the induced degree deficits on $\tau \in \mathcal{T}_{1}$ and $\gamma \in \mathcal{T}_{0}$ are defined as

$$
\Delta \boldsymbol{m}(\tau):=\min _{\tau \subset \sigma} \Delta \boldsymbol{m}(\sigma), \quad \boldsymbol{\Delta} \boldsymbol{m}(\gamma):=\min _{\gamma \in \sigma} \Delta \boldsymbol{m}(\sigma) .
$$

Let $R:=\mathbb{R}[s, t]$ be the polynomial ring with coefficients in $\mathbb{R}$. We define $\mathcal{P}_{m_{1} m_{2}} \equiv \mathcal{P}_{\left(m_{1}, m_{2}\right)} \subset R$ as the $\mathbb{R}$-linear vector space of polynomials of bi-degree at most $\left(m_{1}, m_{2}\right)$. If any of $m_{1}, m_{2}$ are negative, then $\mathcal{P}_{m_{1} m_{2}}:=0$.

A second ingredient needed for defining smooth splines on $\mathcal{T}$ is a smoothness distribution on $\mathcal{T}$, i.e., non-negative integers assigned to the interior edges of $\mathcal{T}$ to 
indicate the desired order of smoothness across them. We represent the smoothness distribution in the form of the following map:

$$
\begin{aligned}
\boldsymbol{r}: & \stackrel{\circ}{\mathcal{T}}_{1} \rightarrow \mathbb{Z}_{\geq 0}, \\
& \tau \mapsto \boldsymbol{r}(\tau),
\end{aligned}
$$

such that $\tau, \tau^{\prime} \in h \stackrel{\circ}{\mathcal{T}}_{1}$ (or both in ${ }^{v} \stackrel{\circ}{\mathcal{T}}_{1}$ ) and $\tau \cap \tau^{\prime} \neq \emptyset \Rightarrow \boldsymbol{r}(\tau)=\boldsymbol{r}\left(\tau^{\prime}\right)$.

As noted above, the map $\boldsymbol{r}$ will help us define the smoothness across all interior edges, i.e., for $\tau \in \stackrel{\circ}{\mathcal{T}}_{1}$, splines will be required to be at least $C^{r(\tau)}$ smooth across $\tau$. For $\gamma \in \stackrel{\circ}{\mathcal{T}_{0}}$ such that $\{\gamma\}=\tau_{h} \cap \tau_{v},\left(\tau_{h}, \tau_{v}\right) \in h \stackrel{\circ}{\mathcal{T}}_{1} \times v \stackrel{\circ}{\mathcal{T}}_{1}$, the smoothness in horizontal and vertical directions is defined, respectively, as

$$
\boldsymbol{r}_{\gamma, h}=\boldsymbol{r}\left(\tau_{v}\right), \quad \boldsymbol{r}_{\gamma, v}=\boldsymbol{r}\left(\tau_{h}\right) .
$$

Definition 2.2 (Spline space) Given a T-mesh $\mathcal{T}$, a degree deficit distribution $\boldsymbol{\Delta m}$, a smoothness distribution $\boldsymbol{r}$ and a bi-degree $\boldsymbol{m} \in \mathbb{Z}_{\geq 0}^{2}$, we define the spline space $\mathcal{R}_{\Delta \boldsymbol{m}, \boldsymbol{m}}^{r}(\mathcal{T})$ as

$$
\mathcal{R}_{\boldsymbol{\Delta} \boldsymbol{m}, \boldsymbol{m}}^{\boldsymbol{r}} \equiv \mathcal{R}_{\boldsymbol{\Delta} \boldsymbol{m}, \boldsymbol{m}}^{r}(\mathcal{T}):=\left\{\begin{array}{cl}
f: & \forall \sigma \in \mathcal{T}_{2},\left.\quad f\right|_{\sigma} \in \mathcal{P}_{\boldsymbol{m}_{\sigma}}=\mathcal{P}_{\boldsymbol{m}-\boldsymbol{\Delta m}(\sigma)}, \\
& \left.\forall \tau \in \stackrel{\circ}{\mathcal{T}}_{1}, \quad f \text { is } C^{\boldsymbol{r}(\tau)} \text { across } \tau\right\}
\end{array}\right.
$$

Example 2.3 A T-mesh is shown in Fig. 1a, and example degree-deficits on its faces are shown in Fig. 1b. The set $\mathcal{D}$ is given by

$$
\mathcal{D}=\{(0,0),(1,1),(2,2)\},
$$

and the sequence $\Delta \boldsymbol{m}_{i}$ can be chosen to be

$$
(0,0)=\Delta m_{0}<(1,1)=\Delta m_{1}<(2,1)=\Delta m_{2}<(2,2)=\Delta m_{3} .
$$

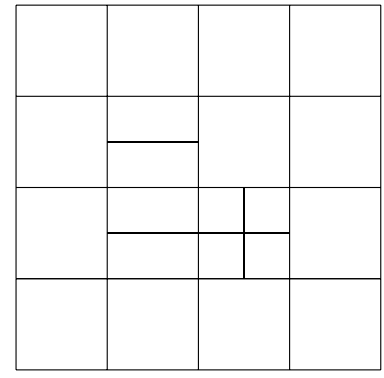

a

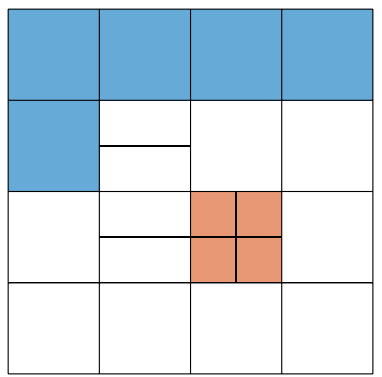

b

Fig. 1 Figure a shows an example T-mesh. Figure b shows a color-coded representation of the degreedeficits on it; the deficit on the white faces is $(2,2)$, on the blue faces is $(1,1)$, and on red faces is $(0,0)$ 
Clearly, the above choice of the sequence is not unique. We could have alternatively chosen the shorter sequence:

$$
(0,0)=\Delta m_{0}<(1,1)=\Delta m_{1}<(2,2)=\Delta m_{2} .
$$

We will employ the following algebraic characterization of smoothness of piecewise-polynomial splines [3].

Lemma $2.4([2,22])$ For $\sigma, \sigma^{\prime} \in \mathcal{T}_{2}$, let $\sigma \cap \sigma^{\prime}=\tau \in \stackrel{\circ}{\mathcal{T}}_{1}$. A piecewise polynomial function equaling $p \in R$ and $p^{\prime} \in R$ on $\sigma$ and $\sigma^{\prime}$, respectively, is at least $r$ times continuously differentiable across $\tau$ if and only if

$$
p-p^{\prime} \in\left(l^{r+1}\right),
$$

where $l \in R$ is a non-zero linear polynomial vanishing on $\tau$.

\subsection{Homogenized problem}

We will translate the problem of investigating the dimension of $\mathcal{R}_{\Delta \boldsymbol{m}, \boldsymbol{m}}^{r}$ to the homogeneous setting $[2,3]$. To this end, let us introduce the ring of bi-homogeneous polynomials $S:=R[u, v]=\mathbb{R}[s, t, u, v]$ which is interpreted as the extension of $R$ by the variables $u$ and $v$ that homogenize $s$ and $t$, respectively. We denote the associated vector space of bi-homogeneous polynomials of bi-degree exactly $\boldsymbol{m}=\left(m_{1}, m_{2}\right) \in \mathbb{Z}_{\geq 0}^{2}$ with $S_{\boldsymbol{m}} \equiv S_{m_{1} m_{2}} \subset S$. If any of $m_{1}, m_{2}$ are negative then $S_{\boldsymbol{m}}:=0$. The ring $S$ is naturally graded by $\mathbb{Z}^{2}, S_{i j} S_{k l}=S_{(i+k)(j+l)}$, and its graded pieces are shifted in the usual manner: $S(-i,-j)_{k l}=S_{(k-i)(l-j)}$.

For convenience, let us define the following notation $s:=(s, t), \boldsymbol{u}:=(u, v)$, and for any tuple $(a, b),(a, b)^{(i, j)}:=a^{i} b^{j}$, for $i, j \in \mathbb{Z}_{\geq 0}$. Using the above, we define the vector space associated to $\square \in \mathcal{T}_{2} \cup \mathcal{T}_{1} \cup \mathcal{T}_{0}$ as

$$
S_{\square}:=\left(\boldsymbol{u}^{\Delta m(\square)}\right)=\boldsymbol{u}^{\Delta m(\square)} S(-\Delta m(\square)) .
$$

In particular, given $\boldsymbol{m} \in \mathbb{Z}_{\geq 0}^{2}$, we denote its $\boldsymbol{m}^{\text {th }}$ graded piece as $S_{\square, \boldsymbol{m}}=$ $\boldsymbol{u}^{\boldsymbol{\Delta} \boldsymbol{m}(\square)} S(-\boldsymbol{\Delta} \boldsymbol{m}(\square))_{\boldsymbol{m}}$. An algebraic characterization of smoothness for bihomogeneous piecewise polynomial functions follows in the vein of Lemma 2.4, and is stated below. The module of bi-homogeneous splines of interest is defined immediately thereafter.

Lemma 2.5 For $\sigma, \sigma^{\prime} \in \mathcal{T}_{2}$, let $\sigma \cap \sigma^{\prime}=\tau \in \stackrel{\circ}{\mathcal{T}}_{1}$. A bi-homogeneous piecewise polynomial function equaling $p \in S$ and $p^{\prime} \in S$ on $\sigma$ and $\sigma^{\prime}$, respectively, is at least $\boldsymbol{r}(\tau)$ times continuously differentiable across $\tau$ if and only if

$$
p-p^{\prime} \in\left(l_{\tau}^{r(\tau)+1}\right),
$$

where $l_{\tau}$ is a non-zero u-homogeneous (resp. v-homogeneous) linear polynomial vanishing on $\tau \in{ }^{v} \mathcal{T}_{1}$ (resp. $\tau \in{ }^{h} \mathcal{T}_{1}$ ). 
Definition 2.6 (Module of bi-homogeneous splines) Given T-mesh $\mathcal{T}$, degree, and smoothness distributions $\boldsymbol{\Delta} \boldsymbol{m}$ and $\boldsymbol{r}$, respectively, we define the module of bi-homogeneous splines $\mathcal{S}_{\Delta \boldsymbol{m}}^{r}(\mathcal{T})$ as

$$
\begin{aligned}
\mathcal{S}_{\Delta \boldsymbol{m}}^{r} \equiv \mathcal{S}_{\boldsymbol{\Delta} \boldsymbol{m}}^{r}(\mathcal{T}):=\left\{f=\sum_{\sigma \in \mathcal{T}_{2}}[\sigma] f_{\sigma}:\right. & \forall \sigma \in \stackrel{\mathcal{T}}{2},, f_{\sigma} \in S_{\sigma}, \\
& \left.\forall \tau \in \stackrel{\circ}{\mathcal{T}}_{1}, \quad \tau=\sigma \cap \sigma^{\prime} \Rightarrow f_{\sigma}-f_{\sigma^{\prime}} \in\left(l_{\tau}^{r(\tau)+1}\right)\right\},
\end{aligned}
$$

where $l_{\tau}$ is the homogenization of the linear polynomial vanishing on $\tau \in \mathcal{T}_{1}$.

Theorem 2.7 Given $\boldsymbol{m} \in \mathbb{Z}_{\geq 0}^{2}$, the $\boldsymbol{m}$-th graded piece of the $S$-module of splines $\mathcal{S}_{\Delta m}^{r}$ is isomorphic to $\mathcal{R}_{\Delta m, m}^{r}$, i.e.,

$$
\operatorname{dim}\left(\mathcal{S}_{\Delta m}^{r}\right)_{m}=\operatorname{dim}\left(\mathcal{R}_{\Delta m, m}^{r}\right)
$$

Proof The proof follows from Lemma 2.5 and Definitions 2.2 and 2.6.

\section{Topological complexes}

In this section, we will describe the tools from homology that we will use for computing the dimension of graded pieces of $\mathcal{S}_{\Delta m}^{r}$ but first let us introduce the relevant notation.

First, we define the ideal:

$$
\mathfrak{L}_{[i]}(-(j, k)) \equiv \mathfrak{L}_{[i]}(-j,-k):=\left\{\begin{array}{ll}
0, & i=\mathfrak{l}+1 \\
\boldsymbol{u}^{\Delta \boldsymbol{m}_{i}} S\left(-\boldsymbol{\Delta} \boldsymbol{m}_{i}-(j, k)\right), & 0 \leq i \leq \mathfrak{l}
\end{array},\right.
$$

and denote $\mathfrak{L}_{[i]} \equiv \mathfrak{L}_{[i]}(0,0)$. Then, from Equation (2.4), we have for $1 \leq i \leq \mathfrak{l}$,

$$
\mathfrak{L}_{[i]}(-j,-k)=\boldsymbol{u}^{\Delta n_{i}} \mathfrak{L}_{[i-1]}\left(-(j, k)-\Delta n_{i}\right) .
$$

We also define the following (shifted) quotient for $1 \leq i \leq \mathfrak{l}+1$,

$$
\mathfrak{M}_{[i]}(-j,-k):=\mathfrak{L}_{[i-1]}(-j,-k) / \mathfrak{L}_{[i]}(-j,-k) .
$$

For $\tau \in \stackrel{\circ}{\mathcal{T}}_{1}$, it will be convenient to set $\Delta_{\tau}:=l_{\tau}^{r(\tau)+1}$, where $l_{\tau}$ is the homogeneous linear polynomial from Lemma 2.5 . We will use $\Delta_{\tau}$ to define the edge and vertex associated ideals $\mathfrak{I}_{\tau}$ and $\mathfrak{I}_{\gamma}$,

$$
\mathfrak{I}_{\tau}:=\left(\Delta_{\tau} \boldsymbol{u}^{\Delta m(\tau)}\right) \subset S_{\tau}, \quad \mathfrak{I}_{\gamma}:=\sum_{\gamma \in \tau}\left(\Delta_{\tau} \boldsymbol{u}^{\Delta m(\tau)}\right) \subset S_{\gamma} .
$$

In general, while $\mathfrak{I}_{\tau}=S_{\tau} \cap\left(\Delta_{\tau}\right)$, we have $\mathfrak{I}_{\gamma}=S_{\gamma} \cap \sum_{\gamma \in \tau}\left(\Delta_{\tau}\right)$ only when $\gamma$ is a crossing vertex, i.e., when $\gamma$ is shared by two horizontal and two vertical edges. 


\subsection{Definitions}

Oriented $i$-cells of $\mathcal{T}$ will generate $S$-modules, and we will index the generators with the respective faces, edges, and vertices of $\mathcal{T}$ : e.g., $[\sigma],[\tau]$, and $[\gamma]$, respectively. We will assume that all oriented 2-cells have been assigned a counterclockwise orientation. For $\tau \in \mathcal{T}_{1}$ with end points $\gamma, \gamma^{\prime} \in \mathcal{T}_{0},[\tau]=\left[\gamma \gamma^{\prime}\right]$, with $\left[\gamma^{\prime} \gamma\right]=-\left[\gamma \gamma^{\prime}\right]$ defining the oppositely oriented edge. Finally, we will consider cellular boundary maps, denoted $\partial$, relative to $\partial \Omega[16]$ :

$$
\partial([\sigma])=\sum_{\tau \in \mathcal{T}_{1}} \varepsilon_{\sigma, \tau}[\tau], \quad \partial([\tau])=\sum_{\gamma \in \stackrel{\circ}{\mathcal{T}}_{0}} \varepsilon_{\tau, \gamma}[\gamma], \quad \partial([\gamma])=0,
$$

where $\varepsilon_{\theta, \phi} \in\{-1,0,+1\}$ denotes if the orientation of the $(n-1)$-cell $[\phi]$ is compatible with that of the $n$-cell $[\theta]$. As is clear from the above equation, we set $[\tau]=0=[\gamma]$ for $\tau, \gamma$ contained in $\partial \Omega$ since only relative homology is of interest here.

Then, for an element $p=\sum_{\sigma}[\sigma] p_{\sigma}$ of the $S$-module $\oplus_{\sigma \in \mathcal{T}_{2}}[\sigma] S_{\sigma}$, its image under the action of $\partial$ will be:

$$
\partial\left(\sum_{\sigma \in \mathcal{T}_{2}}[\sigma] p_{\sigma}\right)=\sum_{\tau \in \stackrel{\mathcal{T}}{1}_{1}}[\tau]\left(\sum_{\sigma \in \mathcal{T}_{2}} \varepsilon_{\sigma, \tau} p_{\sigma}\right) .
$$

It is clear that $\sum_{\sigma} \varepsilon_{\sigma, \tau} p_{\sigma} \in S_{\tau}\left(=\boldsymbol{u}^{\Delta \boldsymbol{m}(\tau)} S\right)$. Therefore, by Lemma 2.5, for $p$ to be in smoothness class $C^{\boldsymbol{r}}$ we require the following:

$$
\forall \tau \in \stackrel{\circ}{\mathcal{T}}_{1}, \quad \sum_{\sigma \in \mathcal{T}_{2}} \varepsilon_{\sigma, \tau} p_{\sigma} \in \mathfrak{I}_{\tau} .
$$

Then, $\mathcal{S}_{\boldsymbol{\Delta} \boldsymbol{m}}^{\boldsymbol{r}}$ contains all splines $f$ (in all bi-degrees) such that their polynomial pieces satisfy the above requirement, with $p_{\sigma}=\left.f\right|_{\sigma}$. In other words, for $\boldsymbol{m} \in \mathbb{Z}_{\geq 0}^{2}$,

$$
\operatorname{dim}\left(\mathcal{R}_{\boldsymbol{\Delta} \boldsymbol{m}, \boldsymbol{m}}^{r}\right)=\operatorname{dim}\left(\mathcal{S}_{\boldsymbol{\Delta} \boldsymbol{m}}^{r}\right)_{\boldsymbol{m}}=\operatorname{dim}(\operatorname{ker}(\bar{\partial}))_{\boldsymbol{m}},
$$

where $\bar{\partial}$, given below, is obtained by composing $\partial$ with the natural quotient map:

$$
\bar{\partial}: \underset{\sigma \in \mathcal{T}_{2}}{\oplus}[\sigma] S_{\sigma} \rightarrow \underset{\tau \in \mathcal{O}_{1}}{\bigoplus_{\circ}}[\tau] S_{\tau} / \mathfrak{I}_{\tau}
$$

\subsection{Degree-deficit-based topological complexes}

In light of the above reasoning, we consider the following chain complex of $S$ modules as the object of our analysis, with the top homology module of $\mathcal{Q}$ equaling $\mathcal{S}_{\Delta m}^{r}$

$\mathcal{Q}: \quad \underset{\sigma \in \mathcal{T}_{2}}{\oplus}[\sigma] S_{\sigma} \longrightarrow \underset{\tau \in \mathcal{T}_{1}}{\oplus^{[}}[\tau] S_{\tau} / \mathfrak{I}_{\tau} \longrightarrow \underset{\gamma \in \stackrel{\circ}{\mathcal{T}}_{0}}{\oplus_{\tau}}[\gamma] S_{\gamma} / \mathfrak{I}_{\gamma} \longrightarrow 0$ 
We analyze $\mathcal{Q}$ by performing a decomposition that untangles the individual contributions from the different degree-deficits from Equation (2.3) to the dimension of $\mathcal{S}_{\Delta m}^{r}$

First, for $\square \in \mathcal{T}_{2} \cup \mathcal{T}_{1} \cup \mathcal{T}_{0}$, we define:

$$
\begin{aligned}
S_{\square,\|i\|} & =S_{\square} /\left(S_{\square} \cap \mathfrak{L}_{[i]}\right) & & \mathfrak{I}_{\square,\|i\|}=\mathfrak{I}_{\square} \cdot S_{\square,\|i\|}, \\
S_{\square,[i]} & =S_{\square,\|i\|} \cap \mathfrak{L}_{[i-1]}, & & \mathfrak{I}_{\square,[i]}=\mathfrak{I}_{\square} \cdot S_{\square,[i]} .
\end{aligned}
$$

We also define the complex $\mathcal{Q}_{[i]}$ as

$$
\underset{\sigma \in \mathcal{T}_{2}}{\oplus}[\sigma] S_{\sigma,[i]} \longrightarrow \underset{\tau \in \mathcal{\mathcal { T }}_{1}}{\oplus^{\circ}}[\tau] S_{\tau,[i]} / \mathfrak{I}_{\tau,[i]} \longrightarrow \underset{\gamma \in \mathcal{\mathcal { T }}_{0}}{\oplus_{\gamma}}[\gamma] S_{\gamma,[i]} / \mathfrak{I}_{\gamma,[i]} \longrightarrow 0,
$$

and the complex $\mathcal{Q}_{\|i\|}$ as

$$
\underset{\sigma \in \mathcal{T}_{2}}{\oplus}[\sigma] S_{\sigma,\|i\|} \longrightarrow \underset{\tau \in \oplus_{\mathcal{T}}}{\bigoplus_{1}}[\tau] S_{\tau,\|i\|} / \mathfrak{I}_{\tau,\|i\|} \longrightarrow \underset{\gamma \in \stackrel{\circ}{\mathcal{T}}_{0}}{\bigoplus_{\gamma}}[\gamma] S_{\gamma,\|i\|} / \mathfrak{I}_{\gamma,\|i\|} \longrightarrow 0
$$

Let us make a few observations about the setup so far and the motivations behind it:

- By construction of these complexes, we have a sequence of complexes:

$$
0 \longrightarrow \mathcal{Q}_{[i]} \longrightarrow \mathcal{Q}_{\|i\|} \longrightarrow \mathcal{Q}_{\|i-1\|} \longrightarrow 0 \text {. }
$$

In other words, the components of $\mathcal{Q}_{[i]}, \mathcal{Q}_{\|i\|}, \mathcal{Q}_{\|i-1\|}$ and the corresponding maps induced form complexes. As we will see in Proposition 3.1, these complexes are exact.

- With regard to the study of $\mathcal{Q}$ from Equation (3.10), it is clear from the above definitions that $\mathcal{Q}_{\|\mathfrak{r}+1\|}=\mathcal{Q}$. We will perform its analysis using the previous sequence of complexes.

- It can be observed that each module in $\mathcal{Q}_{\|0\|}$ is identically zero, thereby implying $\mathcal{Q}_{\|1\|}=\mathcal{Q}_{[1]}$. Therefore, it is only necessary to analyze the complexes:

$$
\mathcal{Q}_{[\mathfrak{r}+1]}, \mathcal{Q}_{[\mathfrak{l}]}, \ldots, \mathcal{Q}_{[2]}, \mathcal{Q}_{[1]} .
$$

- Finally, for a given $\boldsymbol{m} \in \mathbb{Z}_{\geq 0}^{2}$, it can be observed that the dimension of the $\boldsymbol{m}$-th graded piece of the top homology module of $\mathcal{Q}_{[\mathfrak{l}+1]}$ is equal to the dimension of $\mathcal{R}_{\mathbf{0}, \boldsymbol{m}-\boldsymbol{\Delta} \boldsymbol{m} \text { l }}^{\boldsymbol{r}}$, which is the largest uniform-degree spline space contained in $\mathcal{R}_{\Delta \boldsymbol{m}, \boldsymbol{m}}^{r}$. Therefore, in a rough sense, the contributions from the complexes $\mathcal{Q}_{[l]}, \ldots, \mathcal{Q}_{[1]}$ represent the incremental changes in the dimension of $\mathcal{R}_{\mathbf{0}, \boldsymbol{m}-\boldsymbol{\Delta} \boldsymbol{m}_{\mathrm{l}}}^{r}$ that result from the introduction of non-uniformity in bi-degrees. The net effect of these incremental changes on the dimension of $\mathcal{R}_{\mathbf{0}, \boldsymbol{m}-\boldsymbol{\Delta} \boldsymbol{m}_{\mathfrak{l}}}^{r}$ will intuitively bring us close to the quantity of interest, $\operatorname{dim}\left(\mathcal{R}_{\Delta \boldsymbol{m}, \boldsymbol{m}}^{r}\right)=$ $\operatorname{dim}\left(\mathcal{S}_{\Delta \boldsymbol{m}}^{\boldsymbol{r}}\right)_{\boldsymbol{m}}$

Proposition 3.1 For $1 \leq i \leq \mathfrak{l}+1$, the following is a short exact sequence of complexes:

$$
0 \longrightarrow \mathcal{Q}_{[i]} \longrightarrow \mathcal{Q}_{\|i\|} \longrightarrow \mathcal{Q}_{\|i-1\|} \longrightarrow 0
$$


Proof We have to prove that the following is a short exact sequence for all $\square \in$ $\mathcal{T}_{2} \cup \mathcal{T}_{1} \cup \mathcal{T}_{0}$ :

$$
0 \longrightarrow S_{\square,[i]} / \mathfrak{I}_{\square,[i]} \longrightarrow S_{\square,\|i\|} / \mathfrak{I}_{\square,\|i\|} \longrightarrow S_{\square,\|i-1\|} / \mathfrak{I}_{\square,\|i-1\|} \longrightarrow 0,
$$

where $\mathfrak{I}_{\sigma,[i]}:=0$.

Given $i$, if $S_{\square} \subsetneq \mathfrak{L}_{[i-1]}$, then we must have $S_{\square} \subseteq \mathfrak{L}_{[i]}$, which would imply that all spaces of the above complex are identically 0 . Therefore, the only non-trivial case to consider is when $S_{\square} \supseteq \mathfrak{L}_{[i-1]}$. Explicitly, the non-trivial cases to analyze yield the following complexes:

$$
\begin{gathered}
0 \longrightarrow \mathfrak{L}_{[i-1]} / \mathfrak{L}_{[i]} \longrightarrow S_{\sigma} / \mathfrak{L}_{[i]} \longrightarrow S_{\sigma} / \mathfrak{L}_{[i-1]} \longrightarrow 0, \\
0 \rightarrow \mathfrak{L}_{[i-1]} /\left(\mathfrak{I}_{\tau} \cap \mathfrak{L}_{[i-1]}+\mathfrak{L}_{[i]}\right) \longrightarrow S_{\tau} /\left(\mathfrak{I}_{\tau}+\mathfrak{L}_{[i]}\right) \longrightarrow S_{\tau} /\left(\mathfrak{I}_{\tau}+\mathfrak{L}_{[i-1]}\right) \rightarrow 0, \\
0 \rightarrow \mathfrak{L}_{[i-1]} /\left(\mathfrak{I}_{\gamma} \cap \mathfrak{L}_{[i-1]}+\mathfrak{L}_{[i]}\right) \rightarrow S_{\gamma} /\left(\mathfrak{I}_{\gamma}+\mathfrak{L}_{[i]}\right) \rightarrow S_{\gamma} /\left(\mathfrak{I}_{\gamma}+\mathfrak{L}_{[i-1]}\right) \longrightarrow 0 .
\end{gathered}
$$

It is easy to see that $(a)$ is a short exact sequence. The same observation follows for (b) and (c) since, for $\square \in \mathcal{T}_{1} \cup \mathcal{T}_{0}$, by definition we have:

$$
\mathfrak{L}_{[i-1]} \cap\left(\mathfrak{I}_{\square}+\mathfrak{L}_{[i]}\right)=\left(\mathfrak{I}_{\square} \cap \mathfrak{L}_{[i-1]}+\mathfrak{L}_{[i]}\right),
$$

which transforms $(b)$ and $(c)$ into

$$
0 \longrightarrow\left(\mathfrak{I}_{\square}+\mathfrak{L}_{[i-1]}\right) /\left(\mathfrak{I}_{\square}+\mathfrak{L}_{[i]}\right) \longrightarrow S_{\square} /\left(\mathfrak{I}_{\square}+\mathfrak{L}_{[i]}\right) \longrightarrow S_{\square} /\left(\mathfrak{I}_{\square}+\mathfrak{L}_{[i-1]}\right) \longrightarrow 0 .
$$

As stated above, $\mathcal{Q}$ can be studied by studying the complexes $\mathcal{Q}_{[i]}, i=$ $1,2, \ldots, \mathfrak{l}+1$. We do so by analyzing the following short exact sequence of chain complexes for each $i$.

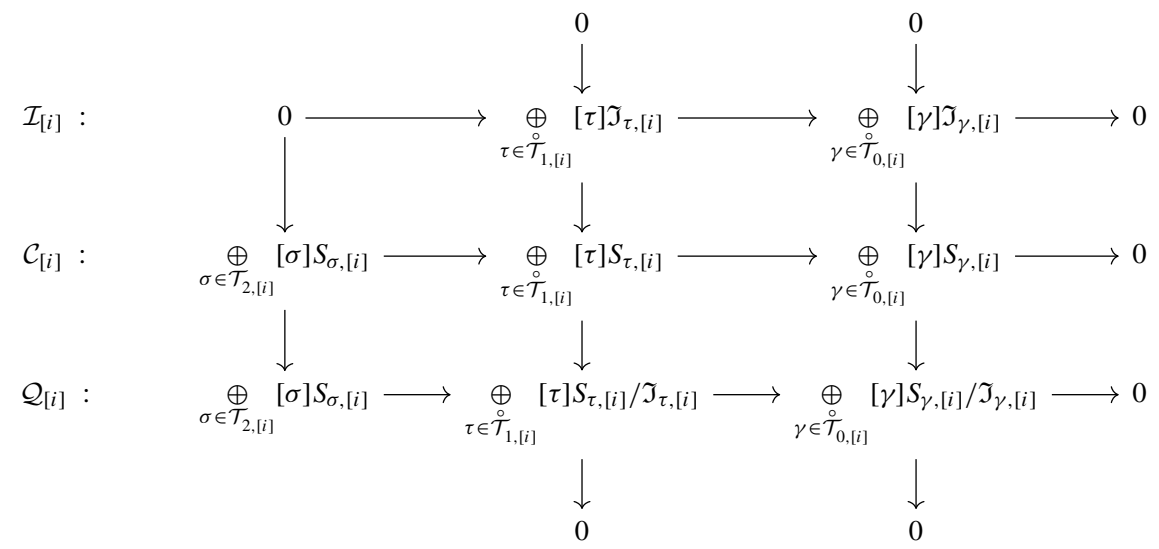


Note that the morphisms above are obtained in the obvious way by composing (restrictions of) $\partial$ with quotient maps. In Equation (3.16), $\mathcal{\mathcal { T }}_{2,[i]}, \stackrel{\circ}{\mathcal{T}}_{1,[i]}$, and $\stackrel{\circ}{\mathcal{T}}_{0,[i]}$ are the active components of the mesh with respect to the index $i$; this notion was hinted at in the proof of Proposition 3.1 and is defined next.

Definition 3.2 (Active T-mesh) For $i=1,2, \ldots, \mathfrak{l}+1$, the active T-mesh $\mathcal{T}_{[i]}$ is composed of

- $\quad \mathcal{T}_{2,[i]} \subseteq \mathcal{T}_{2}$ such that $\sigma \in \mathcal{T}_{2,[i]} \stackrel{\text { def. }}{\Longleftrightarrow} S_{\sigma} \supseteq \mathfrak{L}_{[i-1]} ;$

- $\mathcal{T}_{1,[i]} \subseteq \mathcal{T}_{1}$ such that $\tau \in \mathcal{T}_{1,[i]} \stackrel{\text { def. }}{\Longleftrightarrow} S_{\tau} \supseteq \mathfrak{L}_{[i-1]}$; and,

$-\mathcal{T}_{0,[i]} \subseteq \mathcal{T}_{0}$ such that $\gamma \in \mathcal{T}_{0,[i]} \stackrel{\text { def. }}{\Longleftrightarrow} S_{\gamma} \supseteq \mathfrak{L}_{[i-1]}$.

The domain of this active T-mesh, $\Omega_{[i]}$, is defined to be $\cup_{\sigma \in \mathcal{T}_{2,[i]}} \sigma \subset \mathbb{R}^{2}$.

The symbols for interior edges, vertices, horizontal and vertical edges, etc., are all appended with a subscript of $[i]$ when talking about the active mesh $\mathcal{T}_{[i]}$; see Equation (3.16). Note that "interior" will always mean interior with respect to $\Omega$. It should be noted that $\mathcal{T}_{1,[i]}$ is exactly the set of edges that are contained in $\cup_{\sigma \in \mathcal{T}_{2,[i]}} \partial \sigma$. Similarly, $\mathcal{T}_{0,[i]}$ is exactly the set of vertices that are contained in $\cup_{\tau \in \mathcal{T}_{1,[i]}} \partial \tau$.

Remark 3.3 Note that Equation (2.4) may introduce more active meshes than strictly necessary. However, we choose the degree-deficit sequence in compliance with Equation (2.4) because it simplifies the analysis later on. In particular, the results that are affected by this simplification are Lemmas 6.8 and 6.10 (and those that depend on these lemmas).

Example 3.4 Consider the setup in Example 2.3, and let us choose the shorter sequence of degree deficits provided therein. Then, the associated active meshes with respect to $i=1,2,3$ are shown in Fig. 2. The bottom, middle, and top layers correspond to $\mathcal{T}_{[3]}, \mathcal{T}_{[2]}$, and $\mathcal{T}_{[1]}$, respectively. In other words, the layer corresponding to $\mathcal{T}_{[i]}$ is such that for the faces $\sigma$, edges $\tau$ and vertices $\gamma$ contained in it, we have the containment $S_{\square} \supseteq \mathfrak{L}_{[i-1]}, \square \in\{\sigma, \tau, \gamma\}$.

\subsection{Summary of approach}

Given $\boldsymbol{m} \in \mathbb{Z}_{\geq 0}^{2}$, let $\chi(\mathcal{A})_{\boldsymbol{m}}$ be the Euler characteristic of the $\boldsymbol{m}$-th graded piece of the complex $\overline{\mathcal{A}}: 0 \rightarrow A_{k} \rightarrow A_{k-1} \rightarrow \cdots \rightarrow A_{0} \rightarrow 0$,

$$
\chi(\mathcal{A})_{\boldsymbol{m}}=\sum_{j=0}^{k}(-1)^{j} \operatorname{dim}\left(H_{j}(\mathcal{A})\right)_{\boldsymbol{m}}=\sum_{j=0}^{k}(-1)^{j} \operatorname{dim}\left(A_{j}\right)_{\boldsymbol{m}} .
$$

Then, the Euler characteristic of $\mathcal{Q}=\mathcal{Q}_{\|\mathfrak{l}+1\|}$ helps quantify the homological contribution to the dimension of $\mathcal{S}_{\Delta m}^{r}$. 


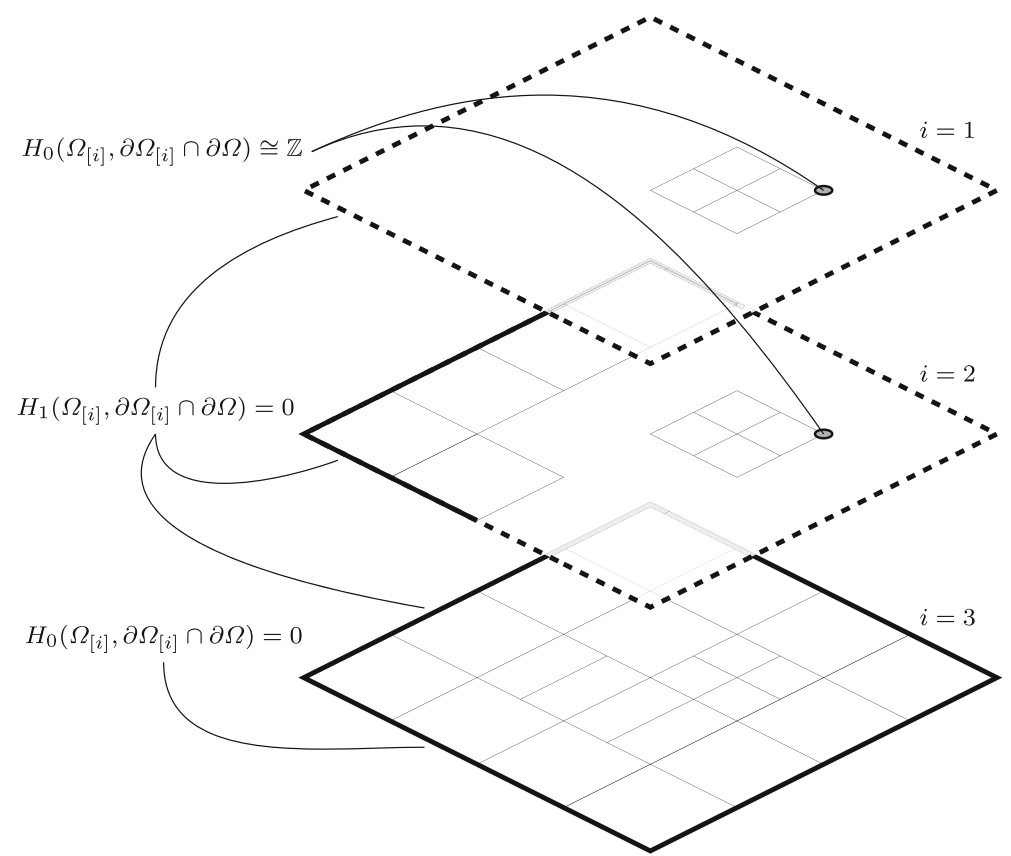

Fig. 2 Active regions of the T-mesh $\mathcal{T}$ from Fig. 1 for different indices $i \in\{1,2,3\}$; see Example 3.4 for reference. Generators of $H_{0}\left(\Omega_{[i]}, \partial \Omega_{[i]} \cap \partial \Omega\right)$ have been shown as gray disks. Boundaries of the meshes have been emphasized in bold and solid lines correspond to the active boundary, i.e., $\partial \Omega \cap \Omega_{[i]}$

Definition 3.5 (Homological contribution to dimension) Given $\boldsymbol{m} \in \mathbb{Z}_{\geq 0}^{2}$, we define the homological contribution to the dimension of $\mathcal{S}_{\Delta \boldsymbol{m}}^{r}$ in bi-degree $\boldsymbol{m}$ as

$$
h_{\Delta \boldsymbol{m}, \boldsymbol{m}}^{\boldsymbol{r}}=\operatorname{dim}\left(\mathcal{S}_{\Delta \boldsymbol{m}}^{\boldsymbol{r}}\right)_{\boldsymbol{m}}-\chi(\mathcal{Q})_{m}
$$

so that we have $\operatorname{dim}\left(\mathcal{S}_{\Delta \boldsymbol{m}}^{r}\right)_{\boldsymbol{m}}=\chi(\mathcal{Q})_{\boldsymbol{m}}+h_{\Delta \boldsymbol{m}, \boldsymbol{m}}^{\boldsymbol{r}}$.

It will be shown in Section 3.4 that the Euler characteristic of $\mathcal{Q}$ is computable exactly using the rightmost expression in Equation (3.17). This leaves only the computation (or estimation) of $h_{\Delta \boldsymbol{m}, \boldsymbol{m}}^{\boldsymbol{r}}$ for the purpose of determining (bounds on) the dimension of $\mathcal{S}_{\boldsymbol{\Delta} \boldsymbol{m}}^{\boldsymbol{r}}$. We approach this task as follows. First, using the short exact sequence from Equation (3.15), we build the long exact sequence of homology modules:

$$
0 \rightarrow H_{2}\left(\mathcal{Q}_{[i]}\right) \rightarrow H_{2}\left(\mathcal{Q}_{\|i\|}\right) \rightarrow H_{2}\left(\mathcal{Q}_{\|i-1\|}\right) \stackrel{\hat{\partial}_{i}}{\rightarrow} H_{1}\left(\mathcal{Q}_{[i]}\right) \rightarrow \cdots \rightarrow H_{0}\left(\mathcal{Q}_{\|i-1\|}\right) \rightarrow 0
$$

with $\hat{\partial}_{1} \equiv 0$. The above implies that

$$
\chi\left(\mathcal{Q}_{\|i\|}\right)_{m}=\chi\left(\mathcal{Q}_{[i]}\right)_{m}+\chi\left(\mathcal{Q}_{\|i-1\|}\right)_{m} .
$$


Summing up the terms for $i=1, \ldots, \mathfrak{l}+1$, we obtain:

$$
\chi(\mathcal{Q})_{m}=\chi\left(\mathcal{Q}_{\|\mathfrak{l}+1\|}\right)_{m}=\sum_{i=1}^{\mathfrak{l}+1} \chi\left(\mathcal{Q}_{[i]}\right)_{m}
$$

since $\mathcal{Q}_{\|0\|}=0$ and $\mathcal{Q}_{\|\mathfrak{l}+1\|}=\mathcal{Q}$. That is, the Euler characteristic of $\mathcal{Q}$ decomposes additively in the above manner. The next results state that a similar additive decomposition does not hold for the 2-homologies.

Lemma 3.6 Given $\boldsymbol{m} \in \mathbb{Z}_{\geq 0}^{2}$, the dimension of the $\boldsymbol{m}^{\text {th }}$ piece of $\mathcal{S}_{\Delta \boldsymbol{m}}^{\boldsymbol{r}}$ can be additively computed as

$$
\operatorname{dim}\left(\mathcal{S}_{\Delta \boldsymbol{m}}^{\boldsymbol{r}}\right)_{\boldsymbol{m}}=\sum_{i=1}^{\mathfrak{l}+1} \operatorname{dim}\left(H_{2}\left(\mathcal{Q}_{[i]}\right)\right)_{\boldsymbol{m}}-\operatorname{dim}\left(\operatorname{im} \hat{\partial}_{i}\right)_{\boldsymbol{m}} .
$$

Proof From Equation (3.18), we have the following exact sequence for all $i \geq 1$,

$$
0 \longrightarrow H_{2}\left(\mathcal{Q}_{[i]}\right) \longrightarrow H_{2}\left(\mathcal{Q}_{\|i\|}\right) \longrightarrow H_{2}\left(\mathcal{Q}_{\|i-1\|}\right) \stackrel{\hat{\partial}_{i}}{\longrightarrow} \operatorname{im} \hat{\partial}_{i} \longrightarrow 0 \text {. }
$$

The above implies that

$$
\operatorname{dim}\left(H_{2}\left(\mathcal{Q}_{\|i\|}\right)\right)_{\boldsymbol{m}}=\operatorname{dim}\left(H_{2}\left(\mathcal{Q}_{\|i-1\|}\right)\right)_{\boldsymbol{m}}+\operatorname{dim}\left(H_{2}\left(\mathcal{Q}_{[i]}\right)\right)_{\boldsymbol{m}}-\operatorname{dim}\left(\operatorname{im} \hat{\partial}_{i}\right)_{\boldsymbol{m}} .
$$

The claim follows upon summing up the terms for $i=1 \ldots, \mathfrak{l}+1$.

Using Lemma 3.6, we can simplify the expression for $\chi(\mathcal{Q})_{m}$,

$$
\begin{aligned}
\chi(\mathcal{Q})_{\boldsymbol{m}} & =\sum_{i=1}^{\mathfrak{l}+1}\left(\operatorname{dim}\left(H_{2}\left(\mathcal{Q}_{[i]}\right)\right)_{\boldsymbol{m}}-\operatorname{dim}\left(H_{1}\left(\mathcal{Q}_{[i]}\right)\right)_{\boldsymbol{m}}+\operatorname{dim}\left(H_{0}\left(\mathcal{Q}_{[i]}\right)\right)_{\boldsymbol{m}}\right) \\
& =\operatorname{dim}\left(\mathcal{S}_{\boldsymbol{\Delta} \boldsymbol{m}}^{\boldsymbol{r}}\right)_{\boldsymbol{m}}+\sum_{i=1}^{\mathfrak{l}+1}\left(\operatorname{dim}\left(\operatorname{im} \hat{\partial}_{i}\right)_{\boldsymbol{m}}-\operatorname{dim}\left(H_{1}\left(\mathcal{Q}_{[i]}\right)\right)_{\boldsymbol{m}}+\operatorname{dim}\left(H_{0}\left(\mathcal{Q}_{[i]}\right)\right)_{\boldsymbol{m}}\right) .
\end{aligned}
$$

A final simplification is afforded by the following assumption.

Assumption 2 All complexes $\mathcal{C}_{[i]}$ are without holes, i.e., $H_{1}\left(\mathcal{C}_{[i]}\right)=0$ for all $i$.

Proposition 3.7 Given $\boldsymbol{m} \in \mathbb{Z}_{\geq 0}^{2}$ and Assumptions 1 and 2, the homological contribution to the dimension can $\bar{b}$ e additively expressed as:

$$
\begin{aligned}
h_{\boldsymbol{\Delta} \boldsymbol{m}, \boldsymbol{m}}^{\boldsymbol{r}} & :=\operatorname{dim}\left(\mathcal{S}_{\boldsymbol{\Delta} \boldsymbol{m}}^{\boldsymbol{r}}\right)_{\boldsymbol{m}}-\chi(\mathcal{Q})_{\boldsymbol{m}}, \\
& =\sum_{i=1}^{\mathfrak{l}+1} \operatorname{dim}\left(H_{0}\left(\mathcal{I}_{[i]}\right)\right)_{\boldsymbol{m}}-\operatorname{dim}\left(H_{0}\left(\mathcal{C}_{[i]}\right)\right)_{\boldsymbol{m}}-\operatorname{dim}\left(\operatorname{im} \hat{\partial}_{i}\right)_{\boldsymbol{m}} .
\end{aligned}
$$

Proof The proof follows from Lemma 3.6 and the diagram in Equation (3.16). Indeed, following Assumption 2, we obtain the long exact sequence of homology 
modules:

$$
0 \longrightarrow H_{1}\left(\mathcal{Q}_{[i]}\right) \longrightarrow H_{0}\left(\mathcal{I}_{[i]}\right) \longrightarrow H_{0}\left(\mathcal{C}_{[i]}\right) \longrightarrow H_{0}\left(\mathcal{Q}_{[i]}\right) \longrightarrow 0
$$

which yields the claim when combined with Equation (3.17).

Therefore, the final problem is the computation (or estimation) of the dimension of im $\hat{\partial}_{i \boldsymbol{m}}$ and the difference in the dimensions of $H_{0}\left(\mathcal{I}_{[i]}\right)_{\boldsymbol{m}}$ and $H_{0}\left(\mathcal{C}_{[i]}\right)_{\boldsymbol{m}}$ for all $i$. The next example shows that in general $\operatorname{dim}\left(\operatorname{im} \hat{\partial}_{i}\right)_{m}$ is not equal to zero.

Example 3.8 Consider the T-mesh in Fig. 3. Assume that the degree deficit on all faces touching the boundary is $(1,1)$, and on the remaining faces is $(0,0)$. Choose the associated degree-deficit sequence to be $\Delta \boldsymbol{m}_{0}=(0,0)<\Delta \boldsymbol{m}_{1}=(1,1)$. Let us ask for $C^{2}$ smoothness across all edges, and let $\boldsymbol{m}=(5,5)$. Then, based on the definitions, it can be checked that:

$$
\operatorname{dim}\left(\mathcal{S}_{\Delta \boldsymbol{m}}^{r}\right)_{\boldsymbol{m}}=81 .
$$

However, we can also compute (using Macaulay2, for instance) that

$$
\operatorname{dim}\left(H_{2}\left(\mathcal{Q}_{[2]}\right)\right)_{\boldsymbol{m}}=81, \quad \operatorname{dim}\left(H_{2}\left(\mathcal{Q}_{[1]}\right)\right)_{\boldsymbol{m}}=1 .
$$

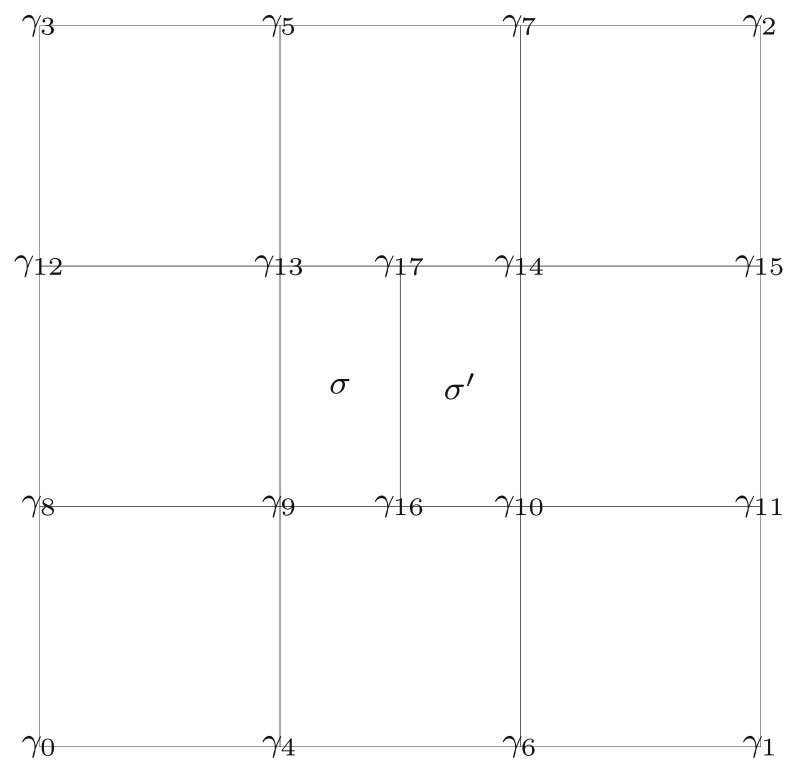

Fig. 3 A mesh that serves to counter the expectation that the spline space dimension can be additively decomposed in the same manner as the Euler characteristic of $\mathcal{Q}$; see Example 3.8 for details 
Thus, $\operatorname{dim}\left(\mathcal{S}_{\boldsymbol{\Delta} \boldsymbol{m}}^{\boldsymbol{r}}\right)_{\boldsymbol{m}} \neq \sum_{i=1}^{2} \operatorname{dim}\left(H_{2}\left(\mathcal{Q}_{[i]}\right)\right)_{\boldsymbol{m}}$. Equivalently, we have that $\operatorname{dim}\left(\operatorname{im} \hat{\partial}_{2}\right)=1$.

In particular, the spline generating $H_{2}\left(\mathcal{Q}_{[2]}\right)$ in degree $\boldsymbol{m}$ is $[\sigma] p+\left[\sigma^{\prime}\right] q$ where

$$
p=t^{5} u(3 s-u)^{3}(9 s-5 u), \quad q=t^{5} u(3 s-2 u)^{3}(9 s-4 u) .
$$

It can be checked that $p-q=27 t^{5} u^{2}(2 s-u)^{3} \in \mathfrak{I}_{\tau}, \tau=\gamma_{16} \gamma_{17}$, and that $p, q \in$ $\mathfrak{I}_{\tau}+(u v)$ where $\tau$ is the edge $\gamma_{13} \gamma_{17}$ or $\gamma_{9} \gamma_{16}$.

\subsection{Dimension formulas for relevant vector spaces}

Before proceeding, we present combinatorial formulas for the dimensions of the different vector spaces that have appeared so far and are relevant for our analysis. In the following, $a_{+}:=\max \{a, 0\}$ for $a \in \mathbb{Z}$. We will also need the bi-smoothness associated to an interior edge $\tau$,

$$
\delta(\tau):=\left\{\begin{array}{ll}
(0, \boldsymbol{r}(\tau)+1), & \tau \in{ }^{h} \stackrel{\circ}{1}_{1} \\
(\boldsymbol{r}(\tau)+1,0), & \tau \in{ }^{v} \mathcal{T}_{1}^{\circ}
\end{array} .\right.
$$

For an interior vertex $\gamma \in \tau_{h} \cap \tau_{v},\left(\tau_{h}, \tau_{v}\right) \in{ }^{h} \stackrel{\circ}{\mathcal{T}}_{1} \times{ }^{v} \mathcal{T}_{1}$, we define $\delta(\gamma):=\delta\left(\tau_{h}\right)+$ $\delta\left(\tau_{v}\right)$.

Proposition 3.9 Let $\tau \in \stackrel{\circ}{\mathcal{T}}_{1,[i]}$, and let $\gamma \in \stackrel{\circ}{\mathcal{T}}_{0,[i]}$ such that $\gamma=\tau_{h} \cap \tau_{v},\left(\tau_{h}, \tau_{v}\right) \in$ $h \stackrel{\circ}{\mathcal{T}}_{1} \times{ }^{v} \stackrel{\circ}{\mathcal{T}}_{1}$. Then, the following hold for $j, k, m, m^{\prime} \in \mathbb{Z}_{\geq 0}, \boldsymbol{m}=\left(m, m^{\prime}\right)$, where for (c) $-(e)$ we assume that $1 \leq i \leq \mathfrak{l}+1$.

$$
\begin{aligned}
& \operatorname{dim}(S(-j,-k))_{\boldsymbol{m}}=(m-j+1)_{+} \times\left(m^{\prime}-k+1\right)_{+} \cdot \\
& \operatorname{dim}\left(\mathfrak{L}_{[i]}(-j,-k)\right)_{\boldsymbol{m}}= \begin{cases}\operatorname{dim}(S(-j,-k))_{\boldsymbol{m}-\boldsymbol{\Delta} \boldsymbol{m}_{i},} & 0 \leq i \leq \mathfrak{l} \\
0, & i=\mathfrak{l}+1\end{cases} \\
& \operatorname{dim}\left(\mathfrak{M}_{[i]}(-j,-k)\right)_{\boldsymbol{m}}=\operatorname{dim}\left(\mathfrak{L}_{[i-1]}(-j,-k)\right)_{\boldsymbol{m}}-\operatorname{dim}\left(\mathfrak{L}_{[i]}(-j,-k)\right)_{\boldsymbol{m}} . \\
& \operatorname{dim}\left(\mathfrak{I}_{\tau,[i]}\right)_{\boldsymbol{m}}=\operatorname{dim}\left(S\left(-\boldsymbol{\delta}(\tau)-\boldsymbol{\Delta} \boldsymbol{m}_{i-1}\right)\right)_{\boldsymbol{m}}-\operatorname{dim}\left(S\left(-\boldsymbol{\delta}(\tau)-\boldsymbol{\Delta} \boldsymbol{m}_{i}\right)\right)_{\boldsymbol{m}} . \\
& \operatorname{dim}\left(\mathfrak{I}_{\gamma,[i]}\right)_{\boldsymbol{m}}=\operatorname{dim}\left(S\left(-\boldsymbol{\delta}\left(\tau_{h}\right)-\boldsymbol{\Delta} \boldsymbol{m}_{i-1}\right)\right)_{\boldsymbol{m}}+\operatorname{dim}\left(S\left(-\boldsymbol{\delta}\left(\tau_{v}\right)-\boldsymbol{\Delta} \boldsymbol{m}_{i-1}\right)\right)_{\boldsymbol{m}} \\
& +\operatorname{dim}\left(S\left(-\boldsymbol{\delta}(\gamma)-\boldsymbol{\Delta} \boldsymbol{m}_{i}\right)\right)_{\boldsymbol{m}}-\operatorname{dim}\left(S\left(-\boldsymbol{\delta}(\gamma)-\boldsymbol{\Delta} \boldsymbol{m}_{i-1}\right)\right)_{\boldsymbol{m}} \\
& -\operatorname{dim}\left(S\left(-\boldsymbol{\delta}\left(\tau_{h}\right)-\boldsymbol{\Delta} \boldsymbol{m}_{i}\right)\right)_{\boldsymbol{m}}-\operatorname{dim}\left(S\left(-\boldsymbol{\delta}\left(\tau_{v}\right)-\boldsymbol{\Delta} \boldsymbol{m}_{i}\right)\right)_{\boldsymbol{m}} .
\end{aligned}
$$

Proof Claims made in parts $(a),(b)$, and $(c)$ follow by definition. Parts $(d)$ and $(e)$ follow from the following exact sequences, respectively:

$$
S\left(-\boldsymbol{\delta}(\tau)-\boldsymbol{\Delta} \boldsymbol{m}_{i}\right) \rightarrow \underset{S\left(-\boldsymbol{\delta}(\tau)-\boldsymbol{\Delta} \boldsymbol{m}_{i-1}\right)}{\stackrel{\oplus}{S\left(-\boldsymbol{\Delta} \boldsymbol{m}_{i}\right)}} \rightarrow \mathfrak{I}_{\tau} \cap \mathfrak{L}_{[i-1]}+\mathfrak{L}_{[i]}
$$




$$
\begin{array}{ccc}
S\left(-\boldsymbol{\delta}\left(\tau_{h}\right)-\boldsymbol{\Delta} \boldsymbol{m}_{i}\right) & & S\left(-\boldsymbol{\delta}\left(\tau_{h}\right)-\boldsymbol{\Delta} \boldsymbol{m}_{i-1}\right) \\
S\left(-\boldsymbol{\delta}(\gamma)-\boldsymbol{\Delta} \boldsymbol{m}_{i}\right) \rightarrow & \begin{array}{c}
\oplus \\
S\left(-\boldsymbol{\delta}\left(\tau_{v}\right)-\boldsymbol{\Delta} \boldsymbol{m}_{i}\right)
\end{array} & \rightarrow\left(-\boldsymbol{\delta}\left(\tau_{v}\right)-\boldsymbol{\Delta} \boldsymbol{m}_{i-1}\right) \\
S\left(-\boldsymbol{\delta}(\gamma)-\boldsymbol{\Delta} \boldsymbol{m}_{i-1}\right) & & S\left(-\boldsymbol{\Delta} \boldsymbol{m}_{i}\right)
\end{array} \mathfrak{I}_{\gamma} \cap \mathfrak{L}_{[i-1]}+\mathfrak{L}_{[i]}
$$

We can simplify the expression in Proposition 3.9(e) for special choices of $\Delta \boldsymbol{m}, \boldsymbol{r}, \boldsymbol{m}$.

Corollary 3.10 Let $1 \leq i \leq \mathfrak{l}$ and $\gamma \in \stackrel{\circ}{\mathcal{T}}_{0,[i]}$ such that $\gamma=\tau_{h} \cap \tau_{v},\left(\tau_{h}, \tau_{v}\right) \in$ $h \stackrel{\circ}{\mathcal{T}}_{1} \times v \stackrel{\circ}{\mathcal{T}}_{1}$. The following statements hold for $\boldsymbol{m}=\left(m, m^{\prime}\right) \in \mathbb{Z}_{\geq 0}$.

(a) If $\boldsymbol{m}-\Delta \boldsymbol{m}_{i}$ is entry-wise greater than or equal to $\left(\boldsymbol{r}\left(\tau_{v}\right), \boldsymbol{r}\left(\tau_{h}\right)\right)$, then

$$
\begin{aligned}
\operatorname{dim}\left(\mathfrak{I}_{\gamma,[i]}\right)_{\boldsymbol{m}} & =\left(m-\Delta m_{(i-1) 1}+1\right) \Delta n_{i 2}+\left(m^{\prime}-\Delta m_{(i-1) 2}+1\right) \Delta n_{i 1}-\Delta n_{i 1} \Delta n_{i 2} \\
& =\operatorname{dim}\left(\mathfrak{M}_{[i]}\right)_{\boldsymbol{m}}=\operatorname{dim}\left(\mathfrak{L}_{[i-1]} / \mathfrak{L}_{[i]}\right)_{\boldsymbol{m}} .
\end{aligned}
$$

(b) If $\boldsymbol{m}-\boldsymbol{\Delta} \boldsymbol{m}_{i-1}$ is entry-wise smaller than or equal to $\left(\boldsymbol{r}\left(\tau_{v}\right), \boldsymbol{r}\left(\tau_{h}\right)\right)$, then

$$
\operatorname{dim}\left(\mathfrak{I}_{\gamma,[i]}\right)_{\boldsymbol{m}}=0
$$

The next result follows from the definition of the modules $\mathfrak{M}_{[i]}(-j,-k)$ and Equation (2.4).

Lemma 3.11 The following hold for $\boldsymbol{m}, \boldsymbol{b} \in \mathbb{Z}_{\geq 0}^{2}$ and $j, k \in \mathbb{Z}_{\geq 0}$, where $1 \leq i \leq \mathfrak{l}+1$ for $(a)$ and $1 \leq i \leq \mathfrak{l}$ for $(b)-(d)$.

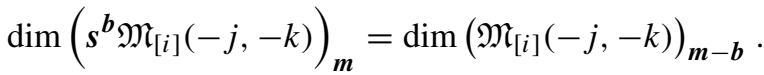

$$
\begin{aligned}
& \operatorname{dim}\left(u \mathfrak{M}_{[i]}(-j,-k)\right)_{\boldsymbol{m}}=\operatorname{dim}\left(u \mathfrak{L}_{[i-1]}(-j,-k)\right)_{\boldsymbol{m}}
\end{aligned}
$$

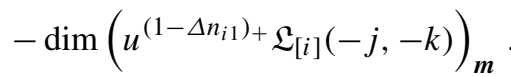

$$
\begin{aligned}
& \operatorname{dim}\left(v \mathfrak{M}_{[i]}(-j,-k)\right)_{\boldsymbol{m}}=\operatorname{dim}\left(v \mathfrak{L}_{[i-1]}(-j,-k)\right)_{\boldsymbol{m}} \\
& -\operatorname{dim}\left(v^{\left(1-\Delta n_{i 2}\right)+} \mathfrak{L}_{[i]}(-j,-k)\right)_{\boldsymbol{m}} \\
& \operatorname{dim}\left(u v \mathfrak{M}_{[i]}(-j,-k)\right)_{m}=0 \text {. }
\end{aligned}
$$

The next result has been adapted from [22] in a form relevant for our analysis; see [22] for its proof.

Proposition 3.12 Consider $\boldsymbol{m}, \boldsymbol{b} \in \mathbb{Z}_{\geq 0}^{2}, \boldsymbol{m}=\left(m, m^{\prime}\right), l$ distinct numbers $a_{1}, \ldots, a_{l} \in \mathbb{R}$, and $d_{1}, \ldots, d_{l} \in \mathbb{Z}_{\geq 0}$. Denote with $f_{k}$ the linear polynomials $t-a_{k} v$. 
Then, we have the following dimension formulae, where $0 \leq i \leq \mathfrak{l}$ for (b) and $1 \leq i \leq \mathfrak{l}+1$ for $(c)$,

$$
\begin{aligned}
& \operatorname{dim}\left(\sum_{k=1}^{l} f_{k}^{d_{k}} S\left(0,-d_{k}\right)\right)_{\boldsymbol{m}}=(m+1) \times \min \left(m^{\prime}+1, \sum_{k=1}^{l} m^{\prime}-d_{k}+1\right) . \\
& \operatorname{dim}\left(\sum_{k=1}^{l} f_{k}^{d_{k}} \mathfrak{L}_{[i]}\left(-\boldsymbol{b}-\left(0, d_{k}\right)\right)\right)_{\boldsymbol{m}}=\operatorname{dim}\left(\sum_{k=1}^{l} f_{k}^{d_{k}} S\left(0,-d_{k}\right)\right)_{\boldsymbol{m}-\boldsymbol{\Delta} \boldsymbol{m}_{i}-\boldsymbol{b}} . \\
& \operatorname{dim}\left(\sum_{k=1}^{l} f_{k}^{d_{k}} \mathfrak{M}_{[i]}\left(-\boldsymbol{b}-\left(0, d_{k}\right)\right)\right)_{\boldsymbol{m}}= \\
& \operatorname{dim}\left(\left(\sum_{k=1}^{l} f_{k}^{d_{k}} \mathfrak{L}_{[i-1]}\left(-\boldsymbol{b}-\left(0, d_{k}\right)\right)\right) / \mathfrak{L}_{[i]}\left(-\boldsymbol{b}-\left(0, d_{k}\right)\right)\right)_{\boldsymbol{m}} .
\end{aligned}
$$

Symmetric claims can be made if the linear polynomials are instead chosen to be $s-a_{k} u$.

\section{Homology of $\mathcal{C}_{[i]}$}

In this section, we collect the main results characterizing the homology of the chain complex $\mathcal{C}_{[i]}$. We will use its following equivalent form that follows from the proof of Proposition 3.1:

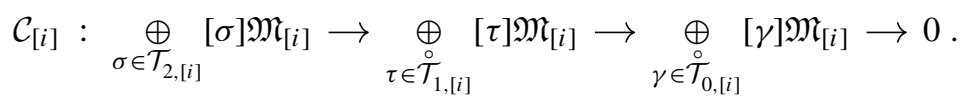

Proposition 4.1 Given $\boldsymbol{m} \in \mathbb{Z}_{\geq 0}^{2}$ and Assumption 1,

$$
\operatorname{dim}\left(H_{2}\left(\mathcal{C}_{[i]}\right)\right)_{\boldsymbol{m}}=\left\{\begin{array}{ll}
\operatorname{dim}\left(\mathfrak{L}_{[\mathfrak{l}]}\right)_{\boldsymbol{m}}, & i=\mathfrak{l}+1 \\
0, & 1 \leq i \leq \mathfrak{l}
\end{array} .\right.
$$

Proof Let $p=\sum_{\sigma}[\sigma] p_{\sigma}, p_{\sigma} \in \mathfrak{L}_{[i-1]}$, be in the kernel of $\partial$, i.e.,

$$
0=\partial(p)=\sum_{\tau \in \stackrel{\circ}{\mathcal{T}}_{1,[i]}}[\tau] \sum_{\sigma \in \mathcal{T}_{2,[i]}} \varepsilon_{\sigma, \tau} p_{\sigma} \Leftrightarrow \forall \tau \in \stackrel{\circ}{\mathcal{T}}_{1,[i]}, \sum_{\sigma \in \mathcal{T}_{2,[i]}} \varepsilon_{\sigma, \tau} p_{\sigma} \in \mathfrak{L}_{[i]} .
$$

If any $\sigma$ and $\sigma^{\prime}$ share an edge $\tau, \varepsilon_{\sigma, \tau}=-\varepsilon_{\sigma^{\prime}, \tau}$. Therefore, if both $\sigma$ and $\sigma^{\prime}$ also belong to $\mathcal{T}_{2,[i]}$, we must have $p_{\sigma}-p_{\sigma^{\prime}} \in \mathfrak{L}_{[i]}$. Then,

- $\quad i=\mathfrak{l}+1$ : Following Assumption 1, all edges in $\stackrel{\circ}{\mathcal{T}}_{1,[\mathfrak{l}+1]}$ are shared by exactly two faces in $\mathcal{T}_{2,[\mathfrak{l}+1]} \equiv \mathcal{T}_{2}$. Therefore, all $p_{\sigma}$ must correspond to the same global polynomial in $\mathfrak{L}_{[\mathfrak{l}]}$ for all faces in $\mathcal{T}_{2}$. 
- $\quad i<\mathfrak{l}+1$ : There exists at least one edge in $\stackrel{\circ}{\mathcal{T}}_{1,[i]}$ that is contained in only one face in $\mathcal{T}_{2,[i]}$. Therefore, all $p_{\sigma}$ must be polynomials in $\mathfrak{L}_{[i]}$, and therefore must be zero in $\mathfrak{L}_{[i-1]} / \mathfrak{L}_{[i]}$.

Definition 4.2 (Number of relative holes in $\Omega_{[i]}$ ) We define $\pi_{[i]}$ to be the number of linearly independent, non-trivial cycles in $\Omega_{[i]}$ relative to $\partial \Omega_{[i]} \cap \partial \Omega$,

$$
\pi_{[i]}:=\operatorname{rank}\left(H_{1}\left(\Omega_{[i]}, \partial \Omega_{[i]} \cap \partial \Omega\right)\right) .
$$

Proposition 4.3 Given $\boldsymbol{m} \in \mathbb{Z}_{\geq 0}^{2}$ and $1 \leq i \leq \mathfrak{l}+1$,

$$
\operatorname{dim}\left(H_{1}\left(\mathcal{C}_{[i]}\right)\right)_{\boldsymbol{m}}=\pi_{[i]} \operatorname{dim}\left(\mathfrak{M}_{[i]}\right)_{\boldsymbol{m}} .
$$

Proof The entire kernel of

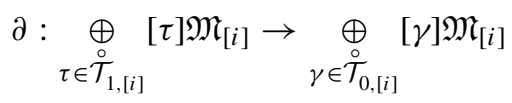

can be generated by ( $\mathbb{R}$-linear combinations of) $c_{k, i}$ of the form

$$
c_{k, i}=p_{i-1} \alpha_{k}+\sum_{\substack{\stackrel{\mathcal{T}}{1,[i]}}}[\tau] p_{\tau, i},
$$

where

- $\quad \alpha_{k}=\sum_{\tau \in \mathcal{T}_{1,[i]}}[\tau] o_{\tau}, o_{\tau} \in \mathbb{Z}$, is a relative cycle, i.e., $\partial \alpha_{k}=0$; and,

- $\quad p_{i-1} \in \mathfrak{L}_{[i-1]}$ and $p_{\tau, i} \in \mathfrak{L}_{[i]}$.

Then, we only need to see how many such $c_{k, i}$ are linearly independent and not nullhomologous. In particular, if $p_{i-1} \notin \mathfrak{L}_{[i]}, c_{k, i}$ is nullhomologous if there exist some $d_{k, i}$ of the form

$$
d_{k, i}=p_{i-1} \beta_{k}+\sum_{\sigma \in \mathcal{T}_{2,[i]}}[\sigma] p_{\sigma, i}
$$

such that $\partial \beta_{k}=\alpha_{k}$, where

- $\beta_{k}=\sum_{\sigma \in \mathcal{T}_{2,[i]}}[\sigma] o_{\sigma}, o_{\sigma} \in \mathbb{Z} ;$ and,

- $p_{\sigma, i} \in \mathfrak{L}_{[i]}$.

Then, $c_{k, i}$ is not nullhomologous in $H_{1}\left(\mathcal{C}_{[i]}\right)$ iff $\alpha_{k}$ is not nullhomologous in $H_{1}\left(\Omega_{[i]}, \partial \Omega_{[i]} \cap \partial \Omega\right)$.

Definition 4.4 (Number of relative connected components in $\Omega_{[i]}$ ) We define $N_{[i]}$ to be the number of connected components in $\Omega_{[i]}$ relative to $\partial \Omega_{[i]} \cap \partial \Omega$,

$$
N_{[i]}:=\operatorname{rank}\left(H_{0}\left(\Omega_{[i]}, \partial \Omega_{[i]} \cap \partial \Omega\right)\right) .
$$


Proposition 4.5 Given $\boldsymbol{m} \in \mathbb{Z}_{\geq 0}^{2}$ and $1 \leq i \leq \mathfrak{l}+1$,

$$
\operatorname{dim}\left(H_{0}\left(\mathcal{C}_{[i]}\right)\right)_{\boldsymbol{m}}=N_{[i]} \operatorname{dim}\left(\mathfrak{M}_{[i]}\right)_{\boldsymbol{m}} .
$$

Proof All $[\gamma] p_{\gamma}, \gamma \in \stackrel{\circ}{\mathcal{T}}_{0,[i]}, p_{\gamma} \in \mathfrak{L}_{[i-1]}$, are in the kernel of $\partial$. Let vertex $\gamma_{0}$, edges $\tau_{1}, \ldots, \tau_{k} \in \stackrel{\circ}{\mathcal{T}}_{1,[i]}, p_{\tau_{l}} \in \mathfrak{L}_{[i-1]}$, and $o_{1}, \ldots, o_{k} \in \mathbb{Z}$ be such that

- $\forall l \in\{1, \ldots, k\}, p_{\tau_{l}}-p_{\gamma} \in \mathfrak{L}_{[i]} ;$ and,

$-\quad[\gamma]=\left[\gamma_{0}\right]+\partial\left(\sum_{l=1}^{k}\left[\tau_{l}\right] o_{l}\right)$.

Assuming $p_{\gamma} \notin \mathfrak{L}_{[i]},[\gamma] p_{\gamma}$ is nullhomologous only if $\gamma$ and $\gamma_{0}$ belong to the same connected component of $\Omega_{[i]}$ and $\gamma_{0} \in \partial \Omega$. Else, $\left[\gamma_{0}\right] p_{\gamma}$ would be a generator of $H_{0}\left(\mathcal{C}_{[i]}\right)$ for the particular connected component of $\Omega_{[i]}$ that it belongs to. Then, for each $p_{\gamma}$, the number of such generators is exactly equal to $N_{[i]}$, and the claim follows.

Example 4.6 Consider the setup in Example 3.4 and Fig. 2. Then, for $\boldsymbol{m}=(5,5)$, it can be verified that:

$$
\begin{gathered}
\operatorname{dim}\left(H_{2}\left(\mathcal{C}_{[i]}\right)\right)_{\boldsymbol{m}}=\left\{\begin{array}{ll}
16, & i=3 \\
0, & i=1,2
\end{array},\right. \\
\operatorname{dim}\left(H_{1}\left(\mathcal{C}_{[i]}\right)\right)_{\boldsymbol{m}}=0, \quad i=1,2,3 \\
\operatorname{dim}\left(H_{0}\left(\mathcal{C}_{[i]}\right)\right)_{\boldsymbol{m}}= \begin{cases}0, & i=3 \\
9, & i=2 \\
11, & i=1\end{cases}
\end{gathered}
$$

\section{The 0-homology of $\mathcal{I}_{[i]}$}

As per our objectives stated at the end of Section 3.3, only the characterization of the 0 -homology of $\mathcal{I}_{[i]}$ remains, and we collect the associated results in this section. Similarly to the previous section, we will do so keeping in mind the simplified form of $\mathcal{I}_{[i]}$ that follows from Proposition 3.1,

$$
\mathcal{I}_{[i]}: 0 \rightarrow \underset{\tau \in \stackrel{\mathcal{T}}{1,[i]}_{0}^{\oplus}}{\oplus}[\tau] \mathfrak{I}_{\tau} \cap \mathfrak{L}_{[i-1]}+\mathfrak{L}_{[i]} / \mathfrak{L}_{[i]} \rightarrow \underset{\gamma \in \stackrel{\mathcal{T}}{0,[i]}_{0^{\prime}}}{\oplus}[\gamma] \mathfrak{I}_{\gamma} \cap \mathfrak{L}_{[i-1]}+\mathfrak{L}_{[i]} / \mathfrak{L}_{[i]} \rightarrow 0
$$

We first provide a lower bound on the dimension of $H_{0}\left(\mathcal{I}_{[i]}\right)$ that holds for special choices of $\Delta \boldsymbol{m}, \boldsymbol{r}$ and $\boldsymbol{m}$.

Proposition 5.1 Let $\boldsymbol{m} \in \mathbb{Z}_{\geq 0}^{2}$ and $1 \leq i \leq \mathfrak{l}$.

(a) If $\boldsymbol{m}-\boldsymbol{\Delta} \boldsymbol{m}_{i}$ is entry-wise greater than or equal to $\left(\boldsymbol{r}_{\gamma, h}, \boldsymbol{r}_{\gamma, v}\right)$ for each $\gamma \in$ $\stackrel{\circ}{\mathcal{T}}_{0,[i]}$, then

$$
\operatorname{dim}\left(H_{0}\left(\mathcal{I}_{[i]}\right)\right)_{\boldsymbol{m}} \geq \operatorname{dim}\left(H_{0}\left(\mathcal{C}_{[i]}\right)\right)_{\boldsymbol{m}}
$$


In particular, the map from $H_{0}\left(\mathcal{I}_{[i]}\right)_{\boldsymbol{m}}$ to $H_{0}\left(\mathcal{C}_{[i]}\right)_{\boldsymbol{m}}$ in Equation (3.18) is a surjection and $H_{0}\left(\mathcal{Q}_{[i]}\right)_{m}$ vanishes.

(b) If $\boldsymbol{m}-\boldsymbol{\Delta} \boldsymbol{m}_{i-1}$ is entry-wise smaller than or equal to $\left(\boldsymbol{r}_{\gamma, h}, \boldsymbol{r}_{\gamma, v}\right)$ for each $\gamma \in$ $\stackrel{\circ}{\mathcal{T}}_{0,[i]}$, then

$$
\operatorname{dim}\left(H_{0}\left(\mathcal{I}_{[i]}\right)\right)_{\boldsymbol{m}}=0 .
$$

In particular, the map from $H_{0}\left(\mathcal{C}_{[i]}\right)_{m}$ to $H_{0}\left(\mathcal{Q}_{[i]}\right)_{m}$ in Equation (3.18) is an isomorphism and $H_{1}\left(\mathcal{Q}_{[i]}\right)_{m}$ vanishes.

\section{Proof}

(a) If $\boldsymbol{m}-\boldsymbol{\Delta} \boldsymbol{m}_{i}$ is entry-wise greater than or equal to $\left(\boldsymbol{r}_{\gamma, h}, \boldsymbol{r}_{\gamma, v}\right)$, then Corollary 3.10 implies that the $\boldsymbol{m}^{\text {th }}$ graded piece of $\oplus_{\gamma \in \stackrel{\mathcal{T}}{0,[i]}}[\gamma] S_{\gamma,[i]} / \mathfrak{I}_{\gamma,[i]}$ vanishes and so does $H_{0}\left(\mathcal{Q}_{[i]}\right)_{\boldsymbol{m}}$.

(b) If $\boldsymbol{m}-\boldsymbol{\Delta} \boldsymbol{m}_{i-1}$ is entry-wise smaller than or equal to $\left(\boldsymbol{r}_{\gamma, h}, \boldsymbol{r}_{\gamma, v}\right)$, then Corollary 3.10 implies that the $\boldsymbol{m}^{\text {th }}$ graded piece of $\oplus_{\gamma \in \stackrel{\circ}{\mathcal{T}},[i]}[\gamma] \mathfrak{I}_{\gamma,[i]}$ vanishes and so does $H_{0}\left(\mathcal{I}_{[i]}\right)_{\boldsymbol{m}}$.

The claims then follow from Equation (3.18).

Let us define the graded multiplication map $\phi_{[i], \gamma}$ for $\gamma \in \stackrel{\circ}{\mathcal{T}}_{0,[i]}$,

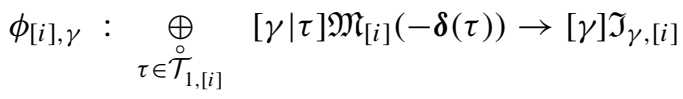

$$
\begin{aligned}
& {[\gamma \mid \tau] p \mapsto[\gamma] p \Delta_{\tau},}
\end{aligned}
$$

where $[\gamma \mid \tau]$ is a half-edge element, with $[\gamma \mid \tau]:=0$ when $\varepsilon_{\tau, \gamma}=0$ or when $\gamma \in \partial \Omega$.

Lemma 5.2 The map $\phi_{[i], \gamma}, 1 \leq i \leq \mathfrak{l}+1$, is surjective.

Proof The claim follows from the isomorphism $\mathfrak{I}_{\tau} \cong S(-\Delta \boldsymbol{m}(\tau)-\delta(\tau))$ and the surjective map:

$$
\begin{array}{cc}
\underset{\tau \in \stackrel{\mathcal{T}}{1,[i]}^{\oplus}}{\oplus} & {[\gamma \mid \tau] \mathfrak{I}_{\tau} \cap \mathfrak{L}_{[i-1]} \rightarrow[\gamma] \mathfrak{I}_{\gamma} \cap \mathfrak{L}_{[i-1]}} \\
& {[\gamma \mid \tau] p \mapsto[\gamma] p .}
\end{array}
$$

Define $E_{h,[i]}(\gamma), E_{v,[i]}(\gamma)$ as the sets of horizontal and vertical edges in $\stackrel{\circ}{\mathcal{T}}_{1,[i]}$ that contain $\gamma \in \stackrel{\circ}{\mathcal{T}}_{0,[i]}$, respectively, and let $E_{[i]}(\gamma)=E_{h,[i]}(\gamma) \cup E_{v,[i]}(\gamma)$. Let $P_{[i]}(\gamma)$ be the set that contains edge-pairs $\left(\tau, \tau^{\prime}\right)$ containing $\gamma$, both either in $E_{h,[i]}(\gamma)$ or $E_{v,[i]}(\gamma)$; we identify $\left(\tau, \tau^{\prime}\right)$ with $\left(\tau^{\prime}, \tau\right)$. Note that, depending on the index $i, P_{[i]}(\gamma)$ may be empty, or may contain either one or two elements. When the vertex $\gamma$ is obvious from the context, we will exclude it from the above notation to simplify the reading of the text. 
Proposition 5.3 For $1 \leq i \leq \mathfrak{l}+1$, the kernel of $\phi_{[i], \gamma}$ is generated by relations between parallel and perpendicular half-edges in the following manner,

$\operatorname{ker}\left(\phi_{[i], \gamma}\right)=\sum_{\left(\tau, \tau^{\prime}\right) \in P_{[i]}}\left([\gamma \mid \tau]-\left[\gamma \mid \tau^{\prime}\right]\right) \mathfrak{M}_{[i]}(-\delta(\tau))+\sum_{\substack{\tau \in E_{v,[i]} \\ \tau^{\prime} \in E_{h,[i]}}}\left([\gamma \mid \tau] \Delta_{\tau^{\prime}}-\left[\gamma \mid \tau^{\prime}\right] \Delta_{\tau}\right) \mathfrak{M}_{[i]}(-\delta(\gamma))$.

Proof With respect to the T-mesh $\mathcal{T}_{[i]}$, let $\gamma$ be a crossing vertex. (The proof for when $\gamma$ is a T-junction will proceed analogously.) Let $\tau_{1}, \tau_{2} \in E_{h,[i]}, \tau_{3}, \tau_{4} \in E_{v,[i]}$, and $P_{[i]}=\left\{\left(\tau_{1}, \tau_{2}\right),\left(\tau_{3}, \tau_{4}\right)\right\}$, and let $p_{j} \in \mathfrak{M}_{[i]}\left(-\delta\left(\tau_{j}\right)\right)$. Then,

$$
\begin{aligned}
\phi_{[i], \gamma}\left(\sum_{j=1}^{4}\left[\gamma \mid \tau_{j}\right] p_{j}\right) & =[\gamma] \sum_{j=1}^{4} p_{j} \Delta_{\tau_{j}}, \\
& =[\gamma]\left(p_{1}+p_{2}\right) \Delta_{\tau_{1}}+[\gamma]\left(p_{3}+p_{4}\right) \Delta_{\tau_{3}},
\end{aligned}
$$

where $\Delta_{\tau_{1}}=\Delta_{\tau_{2}}$, and $\Delta_{\tau_{3}}=\Delta_{\tau_{4}}$, and $\Delta_{\tau_{1}}$ and $\Delta_{\tau_{3}}$ are relatively prime. Therefore, the kernel of the map is generated by:

$$
\left(\left[\gamma \mid \tau_{1}\right]-\left[\gamma \mid \tau_{2}\right]\right) p_{12}, \quad\left(\left[\gamma \mid \tau_{3}\right]-\left[\gamma \mid \tau_{4}\right]\right) p_{34}, \quad\left(\left[\gamma \mid \tau_{h}\right] \Delta_{\tau_{v}}-\left[\gamma \mid \tau_{v}\right] \Delta_{\tau_{h}}\right) p_{h v},
$$

where,

$$
p_{12} \in \mathfrak{M}_{[i]}\left(-\delta\left(\tau_{1}\right)\right), p_{34} \in \mathfrak{M}_{[i]}\left(-\delta\left(\tau_{3}\right)\right), \quad p_{h v} \in \mathfrak{M}_{[i]}(-\delta(\gamma)) .
$$

Using $\phi_{[i], \gamma}$, we can define a map $\phi_{[i]}$ as

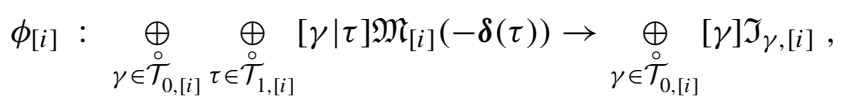

with kernel,

$$
\operatorname{ker}\left(\phi_{[i]}\right)=\sum_{\gamma \in \mathcal{\mathcal { T }}_{0,[i]}} \operatorname{ker}\left(\phi_{[i], \gamma}\right)
$$

Next, let us consider the diagram

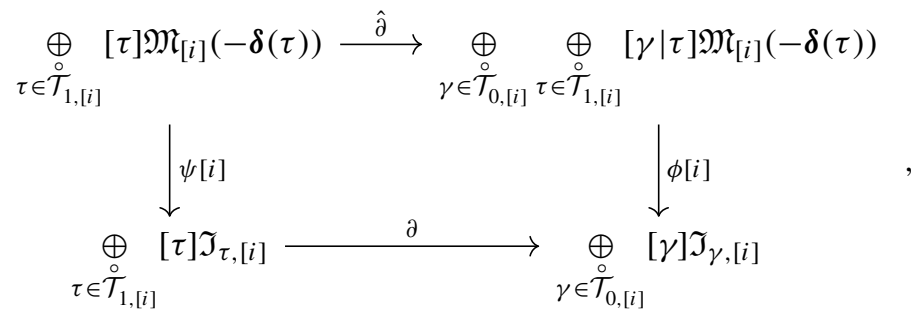

where the maps $\tilde{\partial}$ and $\partial$ are the restrictions of the following maps to the active Tmesh,

$$
\tilde{\partial}:[\tau] \mapsto \sum_{\gamma} \varepsilon_{\tau, \gamma}[\gamma \mid \tau], \quad \partial:[\tau] \mapsto \sum_{\gamma} \varepsilon_{\tau, \gamma}[\gamma]
$$


and the graded map $\psi_{[i]}$ is defined as

$$
\psi_{[i]}:[\tau] \mapsto[\tau] \Delta_{\tau} .
$$

Lemma 5.4 The map $\psi_{[i]}, 1 \leq i \leq \mathfrak{l}+1$, is surjective.

Proof The claim follows from the isomorphism $\mathfrak{I}_{\tau} \cong S(-\Delta m(\tau)-\delta(\tau))$.

Lemma 5.5 The zero homology of $\mathcal{I}_{[i]}, 1 \leq i \leq \mathfrak{l}+1$, is isomorphic to the following quotient of modules generated by half-edges of $\mathcal{T}_{[i]}$,

$$
H_{0}\left(\mathcal{I}_{[i]}\right) \cong \underset{\gamma \in \stackrel{\circ}{\mathcal{T}}_{0,[i]}^{\oplus}}{\underset{\oplus}{\oplus}} \underset{\stackrel{\circ}{\mathcal{T}}_{1,[i]}}{ }[\gamma \mid \tau] \mathfrak{M}_{[i]}(-\boldsymbol{\delta}(\tau)) /\left(\operatorname{ker}\left(\phi_{[i]}\right)+\tilde{\partial}\left(\underset{\tau \in \stackrel{\circ}{\mathcal{T}}_{1,[i]}^{\oplus}}{\oplus}[\tau] \mathfrak{M}_{[i]}(-\boldsymbol{\delta}(\tau))\right)\right) .
$$

Proof The diagram in Equation (5.4) commutes. Indeed,

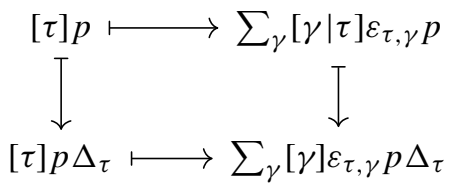

Then, the result follows from surjectivities of $\phi_{[i]}$ and $\psi_{[i]}$ (Lemmas 5.2 and 5.4, respectively), and surjectivity of the induced morphism:

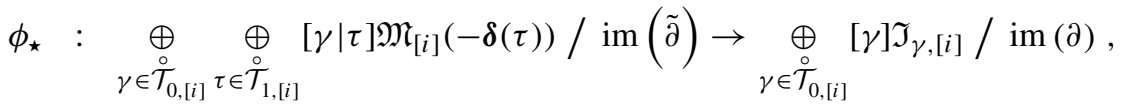

$$
\begin{aligned}
& \sum_{\gamma} \sum_{\tau}[\gamma \mid \tau] p_{\gamma \tau}+\operatorname{im}(\tilde{\partial}) \mapsto \phi_{[i]}\left(\sum_{\gamma} \sum_{\tau}[\gamma \mid \tau] p_{\gamma \tau}\right)+\operatorname{im}(\partial) \text {. }
\end{aligned}
$$

Indeed, the kernel of $\phi_{\star}$ is exactly $\operatorname{ker}\left(\phi_{[i]}\right)+\operatorname{im}(\tilde{\partial}) / \operatorname{im}(\tilde{\partial})$ and we have the isomorphism:

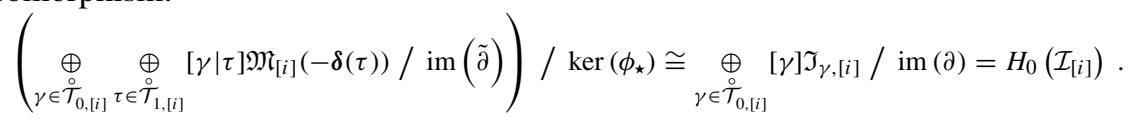

Before proceeding, we first introduce the concept of maximal segments for the T-mesh $\mathcal{T}_{[i]}$. This will help us further simplify the half-edge-based description of $H_{0}\left(\mathcal{I}_{[i]}\right)$ from Proposition 5.5.

Definition 5.6 (Active maximal segments) Given index $i \in\{1, \ldots, \mathfrak{l}+1\}$, the set of active horizontal (resp. vertical) maximal segments ${ }^{h} \mathrm{MS}_{[i]}$ (resp. ${ }^{v} \mathrm{MS}_{[i]}$ ) is the set containing maximal connected unions of edges in ${ }^{h} \mathcal{T}_{1,[i]}$ (resp. $\left.{ }^{v} \mathcal{T}_{1,[i]}\right)$. 
The set of all active maximal segments will be denoted by $\operatorname{MS}_{[i]}=$ ${ }^{h} \mathrm{MS}_{[i]} \cup{ }^{v} \mathrm{MS}_{[i]}$, while the set of active maximal segments that do not intersect the boundary will be denoted by $\mathrm{MS}_{[i]}$; with some abuse of notation, we will refer to these maximal segments as "interior maximal segments." By definition of the smoothness distribution, we can unambiguously define $\delta(\rho)=\delta(\tau)$ and $\Delta_{\rho}=\Delta_{\tau}$ for any edge $\tau \subseteq \rho \in \mathrm{M}_{[i]}$.

Proposition 5.7 The zero homology of $\mathcal{I}_{[i]}, 1 \leq i \leq \mathfrak{l}+1$, is isomorphic to the following quotient of modules generated by active maximal segments of $\mathcal{T}_{[i]}$,

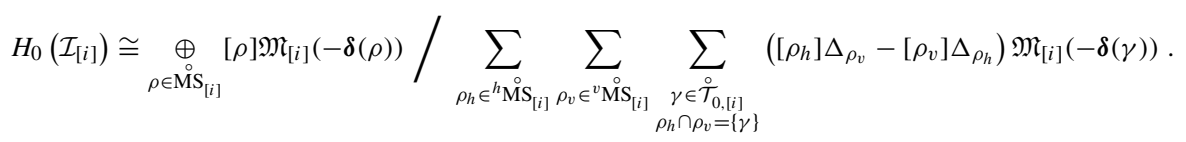

Proof Using Equation (5.3) and Lemma 5.5, we define:

$$
\begin{aligned}
& K^{\prime}=\sum_{\tau \in \mathcal{\mathcal { T }}_{1,[i]}}\left(\sum_{\gamma} \varepsilon_{\tau, \gamma}[\gamma \mid \tau]\right) \mathfrak{M}_{[i]}(-\boldsymbol{\delta}(\tau))+\sum_{\gamma \in \mathcal{\mathcal { T }}_{0,[i]}} \sum_{\left(\tau, \tau^{\prime}\right) \in P_{[i]}}\left([\gamma \mid \tau]-\left[\gamma \mid \tau^{\prime}\right]\right) \mathfrak{M}_{[i]}(-\boldsymbol{\delta}(\tau)), \\
& K=K^{\prime}+\sum_{\substack{\dot{\mathcal{T}}_{0,[i]} \\
\sum_{\tau \in E_{v,[i]}}}}\left([\gamma \mid \tau] \Delta_{\tau^{\prime},[i]}-\left[\gamma \mid \tau^{\prime}\right] \Delta_{\tau}\right) \mathfrak{M}_{[i]}(-\delta(\gamma)) \text {, }
\end{aligned}
$$

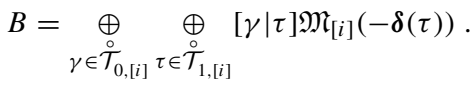

The first term of $K^{\prime}$ corresponds to relations yielding the identification of $[\gamma \mid \tau]$ with $[\tau]$. The second term of $K^{\prime}$ corresponds to relations yielding the identification of $[\gamma \mid \tau]$ with $\left[\gamma \mid \tau^{\prime}\right]$ whenever $\tau, \tau^{\prime} \subset \rho$. Therefore, $K^{\prime}$ leads to the identification of all edges that belong to the same maximal segment $\rho \in \mathrm{MS}_{[i]}$.

Keeping the above in mind, and since $B / K \cong\left(B / K^{\prime}\right) /\left(K / K^{\prime}\right)$, we take the quotient with $K^{\prime}$. The required description is obtained by noticing that, since $[\gamma \mid \tau]=0$ if $\gamma \in \partial \Omega$, terms corresponding to $[\rho]$ must be zero in the quotient for all active maximal segments that intersect $\partial \Omega$.

\section{Bounds on the dimension of $\mathcal{S}_{\Delta m}^{r}$}

We will use Proposition 3.7 in this section to provide upper and lower bounds on the dimensions of graded pieces of $\mathcal{S}_{\boldsymbol{\Delta} \boldsymbol{m}}^{\boldsymbol{r}}$. Some of the results presented here will assume that the condition of sufficiency in Proposition 5.1(a) is satisfied. Therefore, for the sake of convenience, we define the following assumption so that we can refer to it later. 
Assumption 3 Given $\boldsymbol{m} \in \mathbb{Z}_{\geq 0}^{2}$, the degree and smoothness distributions are such that $\boldsymbol{m}-\boldsymbol{\Delta} \boldsymbol{m}_{\mathfrak{l}}$ is entry-wise greater than or equal to $\left(\boldsymbol{r}_{\gamma, h}, \boldsymbol{r}_{\gamma, v}\right)$ for each $\gamma \in \stackrel{\circ}{\mathcal{T}}_{0}$. In particular, this means that the same is true for $\boldsymbol{m}-\Delta \boldsymbol{m}_{i}$ for all $\boldsymbol{i} \leq \mathfrak{l}$.

Theorem 6.1 (Lower bound for general smoothness distributions) Given $\boldsymbol{m} \in \mathbb{Z}_{\geq 0}^{2}$ and Assumptions 1 and 2, the following lower bound on the spline space dimension holds:

$$
\operatorname{dim}\left(\mathcal{S}_{\Delta \boldsymbol{m}}^{\boldsymbol{r}}\right)_{\boldsymbol{m}} \geq \chi(\mathcal{Q})_{\boldsymbol{m}}-\sum_{i=1}^{\mathfrak{l}+1} N_{[i]} \operatorname{dim}\left(\mathfrak{M}_{[i]}\right)_{\boldsymbol{m}}
$$

Proof The lower bound can be arrived at in exactly the same way as Proposition 3.7 but with a slightly different point of departure. Instead of using the short exact sequence in Equation (3.15), we embed the complex $\mathcal{Q}$ directly in the short exact sequence $0 \rightarrow \mathcal{I} \rightarrow \mathcal{C} \rightarrow \mathcal{Q} \rightarrow 0$,

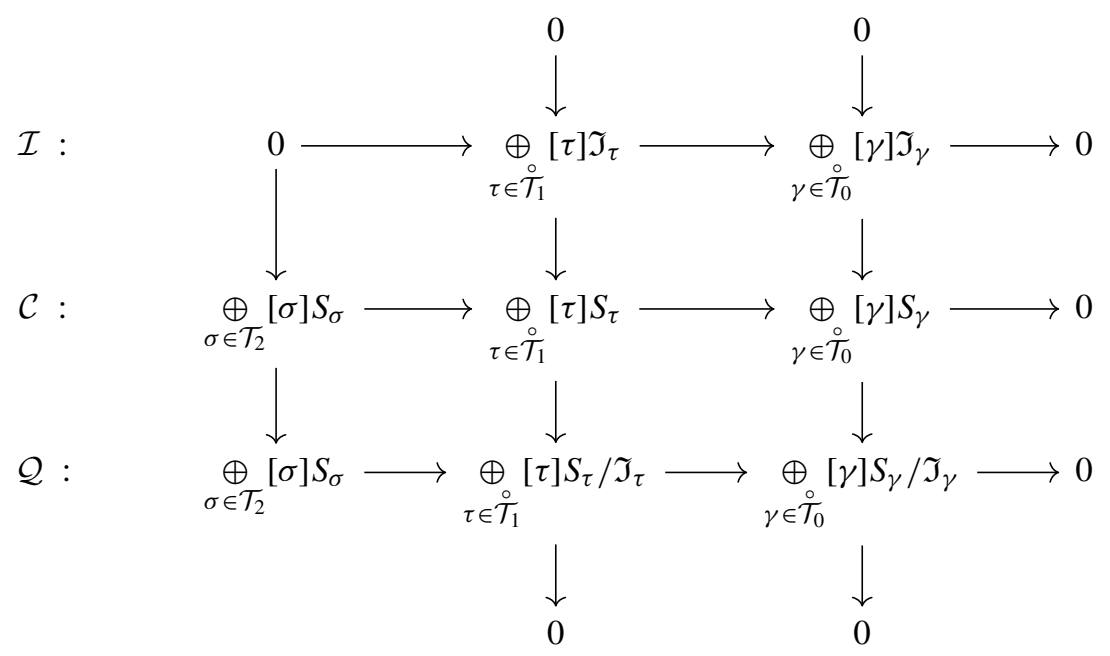

In a manner similar to the proof of Proposition 4.5, it is easy to establish that

$$
\operatorname{dim}\left(H_{0}(\mathcal{C})\right)_{\boldsymbol{m}}=\sum_{i=1}^{\mathfrak{l}+1} \operatorname{dim}\left(H_{0}\left(\mathcal{C}_{[i]}\right)\right)_{\boldsymbol{m}}, \quad \operatorname{dim}\left(H_{1}(\mathcal{C})\right)_{\boldsymbol{m}}=\sum_{i=1}^{\mathfrak{l}+1} \operatorname{dim}\left(H_{1}\left(\mathcal{C}_{[i]}\right)\right)_{\boldsymbol{m}} .
$$

Then, following the same steps as in Section 3.3 but for the diagram above, one can derive the following equation:

$$
\operatorname{dim}\left(\mathcal{S}_{\Delta \boldsymbol{m}}^{\boldsymbol{r}}\right)_{\boldsymbol{m}}=\chi(\mathcal{Q})_{\boldsymbol{m}}+\operatorname{dim}\left(H_{0}(\mathcal{I})\right)_{\boldsymbol{m}}-\operatorname{dim}\left(H_{0}(\mathcal{C})\right)_{\boldsymbol{m}} .
$$

which yields the claimed lower bound since $\operatorname{dim}\left(H_{0}(\mathcal{I})\right)_{\boldsymbol{m}} \geq 0$.

Before presenting a sharper lower bound on the spline space dimension, and with Proposition 3.7 in mind, let us present simple upper bounds on $\operatorname{dim}\left(\operatorname{im} \hat{\partial}_{i}\right)_{\boldsymbol{m}}$. 


\section{Corollary 6.2}

(a) Given $\boldsymbol{m} \in \mathbb{Z}_{\geq 0}^{2}, 1 \leq i \leq \mathfrak{l}+1$ and Assumptions 1 and 2,

$$
\begin{aligned}
& \operatorname{dim}\left(\operatorname{im} \hat{\partial}_{i}\right)_{\boldsymbol{m}} \leq \min \left\{\operatorname{dim}\left(H_{2}\left(\mathcal{Q}_{\|i-1\|}\right)\right)_{\boldsymbol{m}},\right. \\
& \left.\operatorname{dim}\left(H_{0}\left(\mathcal{I}_{[i]}\right)\right)_{\boldsymbol{m}}-N_{[i]} \operatorname{dim}\left(\mathfrak{M}_{[i]}\right)_{\boldsymbol{m}}+\operatorname{dim}\left(H_{0}\left(\mathcal{Q}_{[i]}\right)\right)_{\boldsymbol{m}}\right\} .
\end{aligned}
$$

(b) Given $\boldsymbol{m} \in \mathbb{Z}_{\geq 0}^{2}, 1 \leq i \leq \mathfrak{l}+1$ and Assumptions 1-3,

$$
\operatorname{dim}\left(\operatorname{im} \hat{\partial}_{i}\right)_{\boldsymbol{m}} \leq \min \left\{\operatorname{dim}\left(H_{2}\left(\mathcal{Q}_{\|i-1\|}\right)\right)_{\boldsymbol{m}}, \operatorname{dim}\left(H_{0}\left(\mathcal{I}_{[i]}\right)\right)_{\boldsymbol{m}}-N_{[i]} \operatorname{dim}\left(\mathfrak{M}_{[i]}\right)_{\boldsymbol{m}}\right\} .
$$

Proof The claimed upper bounds can be readily derived using Equation (3.18).

Theorem 6.3 (Lower bound for practical smoothness distributions) Given $\boldsymbol{m} \in \mathbb{Z}_{\geq 0}^{2}$ and Assumptions 1-3, the Euler characteristic of the $\boldsymbol{m}^{\text {th }}$ graded piece of $\mathcal{Q}$ bounds the spline space dimension from below:

$$
\operatorname{dim}\left(\mathcal{S}_{\Delta \boldsymbol{m}}^{r}\right)_{\boldsymbol{m}} \geq \chi(\mathcal{Q})_{m} .
$$

Proof Since the conditions of sufficiency in Proposition 5.1(a) are assumed to be satisfied for all $1 \leq i \leq \mathfrak{l}, H_{0}\left(\mathcal{I}_{[i]}\right)_{\boldsymbol{m}}$ surjects onto $H_{0}\left(\mathcal{C}_{[i]}\right)_{\boldsymbol{m}}$. For $i=\mathfrak{l}+1$, the dimension of $H_{0}\left(\mathcal{I}_{[i]}\right)_{m}$ is trivially bounded from below by $0=\operatorname{dim}\left(H_{0}\left(\mathcal{C}_{[i]}\right)\right)_{\boldsymbol{m}}$. From Corollary 6.2, this implies that

$$
-\operatorname{dim}\left(\operatorname{im} \hat{\partial}_{i}\right)_{\boldsymbol{m}} \geq N_{[i]} \operatorname{dim}\left(\mathfrak{M}_{[i]}\right)_{\boldsymbol{m}}-\operatorname{dim}\left(H_{0}\left(\mathcal{I}_{[i]}\right)\right)_{\boldsymbol{m}} .
$$

The claim follows from Propositions 3.7 and 4.5.

Theorem 6.4 (Upper bound for general smoothness distributions) Given $\boldsymbol{m} \in \mathbb{Z}_{\geq 0}^{2}$ and Assumptions 1-2, the following upper bound on the spline space dimension holds:

$$
\operatorname{dim}\left(\mathcal{S}_{\Delta \boldsymbol{m}}^{\boldsymbol{r}}\right)_{\boldsymbol{m}} \leq \chi(\mathcal{Q})_{\boldsymbol{m}}+\sum_{i=1}^{\mathfrak{l}+1} \operatorname{dim}\left(H_{0}\left(\mathcal{I}_{[i]}\right)\right)_{\boldsymbol{m}}-N_{[i]} \operatorname{dim}\left(\mathfrak{M}_{[i]}\right)_{\boldsymbol{m}}
$$

Proof Since $\operatorname{dim}\left(\operatorname{im} \hat{\partial}_{i}\right)_{m} \geq 0$, the claim follows from Proposition 3.7.

It only remains to derive upper bounds on $\operatorname{dim}\left(H_{0}\left(\mathcal{I}_{[i]}\right)\right)_{m}$ and we do so next. Given a particular $i$, we bound the dimensions of graded pieces of $H_{0}\left(\mathcal{I}_{[i]}\right)$ from above by introducing an ordering on the active interior maximal segments, i.e., on the elements of $\mathrm{MS}_{[i]}$ and by utilizing the representation of $H_{0}\left(\mathcal{I}_{[i]}\right)$ from Proposition 5.7 . 
Definition 6.5 (Ordering of $\mathrm{MS}_{[i]}$ ) Given $i$, let $\xi_{[i]}$ be an ordering on $\mathrm{MS}_{[i]}$, i.e., an injective map from $\mathrm{MS}_{[i]}$ to $\mathbb{N}$. Given $\xi_{[i]}$ and $\rho \in \mathrm{MS}_{[i]}$, define $\Gamma_{[i]}(\rho) \subset \operatorname{MS}_{[i]}$ as the set of maximal segments $\rho^{\prime}$ that intersect $\rho$ non-trivially and such that either $\xi_{[i]}(\rho)>\xi_{[i]}\left(\rho^{\prime}\right)$ or $\rho^{\prime} \cap \partial \Omega \neq \emptyset$.

Hereafter, we will assume that given $i$ the ordering $\xi_{[i]}$ is fixed. We will abuse the notation by using $\rho>\rho^{\prime}$ to mean the same thing as $\xi_{[i]}(\rho)>\xi_{[i]}\left(\rho^{\prime}\right)$. Let us define the modules:

$$
\begin{aligned}
& M_{[i]}:=\underset{\rho \in \operatorname{MS}_{[i]}}{\oplus}[\rho] \mathfrak{M}_{[i]}(-\delta(\rho)), \\
& D_{[i]}:=\sum_{\rho_{h} \in{ }^{h} \mathrm{MS}_{[i]}} \sum_{\rho_{v} \in{ }^{v} \mathrm{MS}_{[i]}} \sum_{\substack{\gamma \in \mathcal{T}_{0,[i]} \\
\rho_{h} \cap \rho_{v}=\{\gamma\}}}\left(\left[\rho_{h}\right] \Delta_{\rho_{v}}-\left[\rho_{v}\right] \Delta_{\rho_{h}}\right) \mathfrak{M}_{[i]}(-\boldsymbol{\delta}(\gamma)) .
\end{aligned}
$$

For $p=\sum_{\rho}[\rho] p_{\rho} \in M_{[i]}$, we define its initial, denoted In $p$, as $\left[\rho^{\prime}\right] p_{\rho^{\prime}}$ if, out of all $\rho$ such that $p_{\rho} \neq 0, \rho^{\prime}$ has the biggest index according to $\xi_{[i]}$.

Lemma 6.6 Given $1 \leq i \leq \mathfrak{l}+1$ and an ordering of $\mathrm{MS}_{[i]}$, the description of $H_{0}\left(\mathcal{I}_{[i]}\right)$ from Proposition 5.7 can be equivalently described as

$$
H_{0}\left(\mathcal{I}_{[i]}\right) \cong M_{[i]} / \text { In } D_{[i]} .
$$

Proof The claim is a standard result; see [23], for example.

Proposition 6.7 Given $\boldsymbol{m} \in \mathbb{Z}_{\geq 0}^{2}$ and $1 \leq i \leq \mathfrak{l}+1$, the following upper bound on the dimension of the $\boldsymbol{m}^{\text {th }}$ graded piece of $H_{0}\left(\mathcal{I}_{[i]}\right)$ holds:

$$
\left.\operatorname{dim}\left(H_{0}\left(\mathcal{I}_{[i]}\right)\right)_{\boldsymbol{m}} \leq \sum_{\rho \in \mathrm{MS}_{[i]}}\left(\operatorname{dim}\left(\mathfrak{M}_{[i]}(-\delta(\rho))\right)_{\boldsymbol{m}}-\operatorname{dim}\left(\sum_{\rho^{\prime} \in \Gamma_{[i]}(\rho)} \Delta_{\rho^{\prime}} \mathfrak{M}_{[i]}\left(-\boldsymbol{\delta}(\rho)-\boldsymbol{\delta}\left(\rho^{\prime}\right)\right)\right)\right)_{\boldsymbol{m}}\right)
$$

Proof From Lemma 6.6, if we can provide a lower bound on the dimension of In $D_{[i]}$, then we can provide an upper bound on $\operatorname{dim}\left(H_{0}\left(\mathcal{I}_{[i]}\right)\right)_{\boldsymbol{m}}$.

Notice that In $D_{[i]}$ is going to be at least partially generated by the initials of its generators. Looking at the generators, for each $\rho \in \mathrm{MS}_{[i]}$, the contributions only come from the $\rho^{\prime} \in \Gamma_{[i]}(\rho)$. The claim follows.

In practical computations, we have observed that if each connected component of $\Omega_{[i]}$ intersects $\partial \Omega$, the upper bound in Proposition 6.7 is usually optimal. Thus, this upper bound is useful for $i=\mathfrak{l}+1$ since $\Omega_{[\mathfrak{l}+1]}=\Omega$. Here, we use "optimal" in the sense of the upper bound coinciding with the exact dimension of $H_{0}\left(\mathcal{I}_{[i]}\right)$ in bi-degree $\boldsymbol{m}$. 
However, for $1 \leq i \leq \mathfrak{l}$, if $\partial \Omega_{[i]}$ does not intersect $\partial \Omega$, it turns out that the upper bound in Proposition 6.7 is a poor estimate. In other words, the initials of the generators of $D_{[i]}$ used in Proposition 6.7 do a bad job of approximating its dimension. Nonetheless, we can significantly improve upon this estimate by systematically adding some new generators to the ones used previously. In particular, doing so will allow us to compute the dimension of $H_{0}\left(\mathcal{I}_{[i]}\right)$ exactly even when there exist connected components of $\Omega_{[i]}$ that do not intersect $\partial \Omega$; see Sections 7 and 8 for examples. In turn, this will enable the use of the sufficient conditions outlined later in Section 7 for computing the exact spline space dimension in many cases.

Following the above comments, for a given maximal segment $\rho \in \mathrm{MS}_{[i]}$, consider the particular connected component of $\Omega_{[i]}$ that $\rho$ belongs to. Let us focus on enlarging the set of generators involving $\rho$ in Equation (6.2). Define $\Upsilon_{[i]}(\rho)$ as the following set of maximal segment pairs:

$$
\Upsilon_{[i]}(\rho):=\left\{\left(\rho_{1}, \rho_{2}\right) \in \operatorname{M}_{[i]} \times \operatorname{MiS}_{[i]}: \rho_{2} \cap \rho \neq \emptyset \neq \rho_{2} \cap \rho_{1}, \boldsymbol{r}(\rho) \geq \boldsymbol{r}\left(\rho_{1}\right), \rho>\rho_{1}\right\} .
$$

Note that in the above definition $\rho$ and $\rho_{1}$ must be parallel and $\rho_{2}$ must be perpendicular to both. In Equation (6.2), the generators of $D_{[i]}$ already contain explicit relations between $[\rho]$ and $\left[\rho_{2}\right]$, and between $\left[\rho_{1}\right]$ and $\left[\rho_{2}\right]$ for $\left(\rho_{1}, \rho_{2}\right) \in \Upsilon_{[i]}(\rho)$. Then, these generators can be manipulated to give a generator involving only $[\rho]$ and $\left[\rho_{1}\right]$.

Lemma 6.8 Given $1 \leq i \leq \mathfrak{l}$ and a maximal segment $\rho \in \operatorname{MS}_{[i]}$, let $\left(\rho_{1}, \rho_{2}\right) \in$ $\Upsilon_{[i]}(\rho)$. Recalling Equation (2.4), define $\boldsymbol{\Delta} \boldsymbol{p}_{\rho}$ as

$$
\Delta \boldsymbol{p}_{\rho}:= \begin{cases}\left(\Delta n_{i 1}, 0\right), & \rho \in{ }^{h} \mathrm{MS}_{[i]}^{j}, \\ \left(0, \Delta n_{i 2}\right), & \rho \in{ }^{v} \mathrm{MS}_{[i]}^{j} .\end{cases}
$$

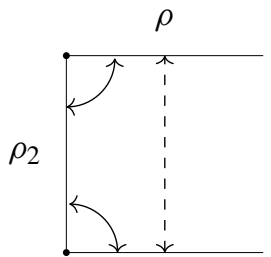

Then, there is a polynomial $\hat{\Delta}$ such that

$$
\boldsymbol{u}^{\boldsymbol{\Delta} \boldsymbol{p}_{\rho}} \Delta_{\rho_{2}}\left([\rho]-\left[\rho_{1}\right] \hat{\Delta}\right) \mathfrak{M}_{[i]}\left(-\boldsymbol{\delta}(\rho)-\boldsymbol{\delta}\left(\rho_{2}\right)-\boldsymbol{\Delta} \boldsymbol{p}_{\rho}\right) \subset D_{[i]} \cdot \rho_{1}
$$

Proof Without loss of generality, let $\rho, \rho_{1} \in{ }^{v} \mathrm{MS}_{[i]}$ and define $r_{\rho \rho_{1}}:=\boldsymbol{r}(\rho)-$ $\boldsymbol{r}\left(\rho_{1}\right) \geq 0$. By definition of $D_{[i]}$, the following are two of its generators:

$$
\begin{aligned}
& \left(\left[\rho_{2}\right] \Delta_{\rho_{1}}-\left[\rho_{1}\right] \Delta_{\rho_{2}}\right) \mathfrak{M}_{[i]}\left(-\delta\left(\rho_{1}\right)-\delta\left(\rho_{2}\right)\right), \\
& \left(\left[\rho_{2}\right] \Delta_{\rho}-[\rho] \Delta_{\rho_{2}}\right) \mathfrak{M}_{[i]}\left(-\delta(\rho)-\delta\left(\rho_{2}\right)\right) .
\end{aligned}
$$

Let $\Delta_{\rho_{1}}=s^{r\left(\rho_{1}\right)+1}$ and $\Delta_{\rho}=(s+a u)^{(r(\rho)+1)}, a \in \mathbb{R}$. We can write $\Delta_{\rho}=\Delta_{\rho_{1}} \hat{\Delta}+$ $\Delta^{\prime}$, where the term with the highest power of $s$ in $\Delta^{\prime}$ is a multiple of $s^{r\left(\rho_{1}\right)} u^{r_{\rho \rho_{1}}+1}$. Then, we can combine the two generators in Equations $\left(\star_{1}\right)$ and $\left(\star_{2}\right)$ to yield:

$$
\left(\star_{2}\right)-\left(\star_{1}\right) \times \hat{\Delta}=\left(\left[\rho_{2}\right] \Delta^{\prime}-[\rho] \Delta_{\rho_{2}}+\left[\rho_{1}\right] \Delta_{\rho_{2}} \hat{\Delta}\right) \mathfrak{M}_{[i]}\left(-\boldsymbol{\delta}(\rho)-\boldsymbol{\delta}\left(\rho_{2}\right)\right) \subset D_{[i]} .
$$


We can further reduce the above relation to

$$
\boldsymbol{u}^{\boldsymbol{\Delta} \boldsymbol{p}_{\rho}} \Delta_{\rho_{2}}\left([\rho]-\left[\rho_{1}\right] \hat{\Delta}\right) \mathfrak{M}_{[i]}\left(-\boldsymbol{\delta}(\rho)-\boldsymbol{\delta}\left(\rho_{2}\right)-\boldsymbol{\Delta} \boldsymbol{p}_{\rho}\right) \subset D_{[i]}
$$

because $\mathfrak{L}_{[i]}\left(-\boldsymbol{\delta}\left(\rho_{2}\right)\right)=\boldsymbol{u}^{\boldsymbol{\Delta} \boldsymbol{n}_{i}} \mathfrak{L}_{[i-1]}\left(-\boldsymbol{\delta}\left(\rho_{2}\right)-\boldsymbol{\Delta} \boldsymbol{n}_{i}\right)$ from Equation (3.2) and $\boldsymbol{\Delta}^{\prime}$ is a multiple of $u$.

Lemma 6.8 shows that, even if $\rho_{2}>\rho$, it may be a part of the contribution that $[\rho]$ makes toward In $D_{[i]}$. Given enough new generators of $D_{[i]}$ of this form, we can go a step further and identify some additional generators. To this end, given a bi-degree $\boldsymbol{m}$, define the set $\Upsilon_{[i]}(\rho, \boldsymbol{m})$ as

$$
\begin{aligned}
\Upsilon_{[i]}(\rho, \boldsymbol{m}):=\left\{\Upsilon \subset \Upsilon_{[i]}(\rho): \sum_{\left(\cdot, \rho^{\prime}\right) \in \Upsilon} \Delta_{\rho^{\prime}} \mathfrak{M}_{[i]}\left(-\boldsymbol{\delta}\left(\rho^{\prime}\right)\right.\right. & \left.-\boldsymbol{\delta}(\rho)-\boldsymbol{\Delta} \boldsymbol{p}_{\rho}\right)_{\boldsymbol{m}} \\
& \left.=\mathfrak{M}_{[i]}\left(-\boldsymbol{\delta}(\rho)-\boldsymbol{\Delta} \boldsymbol{p}_{\rho}\right)_{\boldsymbol{m}}\right\} .
\end{aligned}
$$

Corollary 6.9 Given $\boldsymbol{m}=\left(m, m^{\prime}\right) \in \mathbb{Z}_{\geq 0}^{2}, 1 \leq i \leq \mathfrak{l}$ and $\rho \in \stackrel{\circ}{\mathrm{M}}_{[i]}$, and consider $\Upsilon \subset \Upsilon_{[i]}(\rho)$. Then $\Upsilon \in \Upsilon_{[i]}(\rho, \boldsymbol{m})$ if

$$
\left\{\begin{array}{l}
\sum_{\substack{\rho^{\prime} \\
\left(\cdot, \rho^{\prime}\right) \in \Upsilon}}\left(m-\Delta m_{i 1}-\boldsymbol{r}\left(\rho^{\prime}\right)\right)_{+} \geq m-\Delta m_{i 1}+1, \quad \rho \in{ }^{h} \mathrm{MS}_{[i]}, \\
\sum_{\substack{\rho^{\prime} \\
\left(\cdot, \rho^{\prime}\right) \in \Upsilon}}\left(m^{\prime}-\Delta m_{i 2}-\boldsymbol{r}\left(\rho^{\prime}\right)\right)_{+} \geq m^{\prime}-\Delta m_{i 2}+1, \quad \rho \in{ }^{v} \mathrm{MS}_{[i]} .
\end{array}\right.
$$

Proof The proof follows directly from Proposition 3.12 since $\Delta m_{(i-1) j}+\Delta v_{i j}=$ $\Delta_{i j}, j=1,2$.

Using the above definition, we define the set $\Theta_{[i]}(\rho, \boldsymbol{m})$ as

$$
\begin{aligned}
\Theta_{[i]}(\rho, \boldsymbol{m}):=\left\{\left(\rho_{1}, \rho_{2}\right) \in\right. & \operatorname{MS}_{[i]} \times \stackrel{\circ}{\operatorname{MS}}_{[i]}: \rho_{1} \cap \rho \neq \emptyset \neq \rho_{2} \cap \rho, \boldsymbol{r}\left(\rho_{2}\right) \geq \boldsymbol{r}\left(\rho_{1}\right), \\
& \left.\exists \Upsilon \in \Upsilon_{[i]}(\rho, \boldsymbol{m}), \quad \forall\left(\rho_{3}, \cdot\right) \in \Upsilon, \rho_{2}>\rho_{1}>\rho_{3}\right\} .
\end{aligned}
$$

Given a maximal segment $\rho$ such that $\Upsilon_{[i]}(\rho, \boldsymbol{m})$ is not empty, we can identify further contributions to the initial In $D_{[i], m}$ from maximal segments that intersect $\rho$. The next result elucidates our reasoning. 
Lemma 6.10 Given $\boldsymbol{m} \in \mathbb{Z}_{\geq 0}^{2}, 1 \leq i \leq \mathfrak{l}$ and a fixed maximal segment $\rho$, let $\left(\rho_{1}, \rho_{2}\right) \in \Theta_{[i]}(\rho, \boldsymbol{m})$. Define

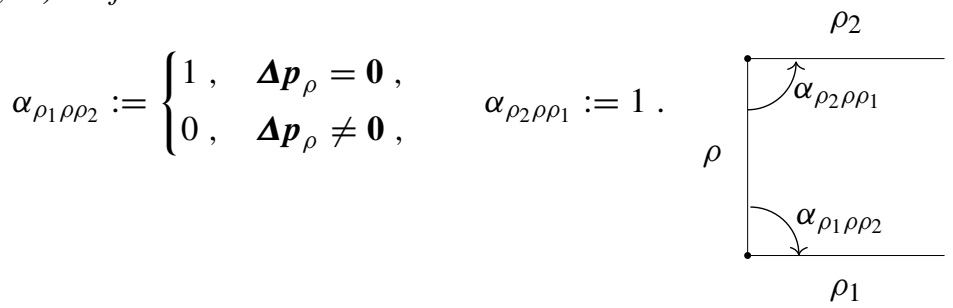

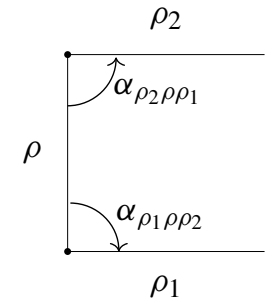

Then, we have

$$
\begin{aligned}
& {\left[\rho_{1}\right] \alpha_{\rho_{1} \rho \rho_{2}} \Delta_{\rho} \mathfrak{M}_{[i]}\left(-\boldsymbol{\delta}\left(\rho_{1}\right)-\boldsymbol{\delta}(\rho)\right)_{\boldsymbol{m}} \subset \text { In } D_{[i], \boldsymbol{m}},} \\
& {\left[\rho_{2}\right] \alpha_{\rho_{2} \rho \rho_{1}} \Delta_{\rho} \mathfrak{M}_{[i]}\left(-\boldsymbol{\delta}\left(\rho_{2}\right)-\boldsymbol{\delta}(\rho)\right)_{\boldsymbol{m}} \subset \text { In } D_{[i], \boldsymbol{m}} \text {. }}
\end{aligned}
$$

Proof From Lemma 6.8, there exist $\Upsilon \in \Upsilon_{[i]}(\rho, \boldsymbol{m})$ and polynomials $\eta_{\rho_{3}}$ such that

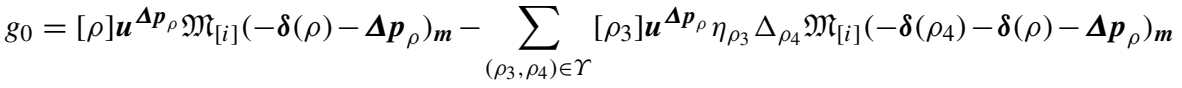

is a relation in $D_{[i], m}$. Then, as in the proof of Lemma 6.8, consider the relations between $\left[\rho_{1}\right]$ and $[\rho]$, and between $\left[\rho_{2}\right]$ and $[\rho]$; denote these with $g_{1}$ and $g_{2}$, respectively. Thereafter, if $\boldsymbol{\Delta} \boldsymbol{p}_{\rho}=\mathbf{0}$, eliminate $[\rho]$ from both $g_{1}$ and $g_{2}$ using $g_{0}$ to get new relations $\bar{g}_{1}$ and $\bar{g}_{2}$; the claim will follow since $\rho_{1}, \rho_{2}>\rho_{3}$ for all $\left(\rho_{3}, \cdot\right) \in \Upsilon$.

If, on the other hand, $\boldsymbol{\Delta} \boldsymbol{p}_{\rho} \neq \mathbf{0}$, combine $g_{1}$ and $g_{2}$ to get a new relation $g_{3}=$ $g_{2}-g_{1} \hat{\Delta}$. For an appropriate choice of $\hat{\Delta}$ (as in the proof of Lemma 6.8), it will be possible to eliminate $[\rho]$ from $g_{3}$, and the initial of $g_{3}$ will thus be a part of the contribution that $\left[\rho_{2}\right]$ makes toward In $D_{[i], \boldsymbol{m}}$.

Finally, we can toss in all the new generators identified in Lemmas 6.8 and 6.10 with the original set of generators in Equation (6.2). Doing so, for $1 \leq i \leq \mathfrak{l}$, we define:

$$
\begin{aligned}
& \sum_{\rho^{\prime} \in \Gamma_{[i]}(\rho)} \Delta_{\rho^{\prime}} \mathfrak{M}_{[i]}\left(-\boldsymbol{\delta}(\rho)-\boldsymbol{\delta}\left(\rho^{\prime}\right)\right)_{\boldsymbol{m}} \\
\mathfrak{D}_{[i], \boldsymbol{m}}^{\rho}:= & +\sum_{\left(\cdot, \rho_{1}\right) \in \Upsilon_{[i]}(\rho)} \boldsymbol{u}^{\boldsymbol{\Delta} \boldsymbol{p}_{\rho}} \Delta_{\rho_{1}} \mathfrak{M}_{[i]}\left(-\boldsymbol{\delta}(\rho)-\boldsymbol{\delta}\left(\rho_{1}\right)-\boldsymbol{\Delta} \boldsymbol{p}_{\rho}\right)_{\boldsymbol{m}} \\
& +\sum_{\left(\rho, \rho_{2}\right) \in \Theta_{[i]}\left(\rho_{3}, \boldsymbol{m}\right)} \alpha_{\rho \rho_{3} \rho_{2}} \Delta_{\rho_{3}} \mathfrak{M}_{[i]}\left(-\boldsymbol{\delta}(\rho)-\boldsymbol{\delta}\left(\rho_{3}\right)\right)_{\boldsymbol{m}} \\
& +\sum_{\left(\rho_{4}, \rho\right) \in \Theta_{[i]}\left(\rho_{5}, \boldsymbol{m}\right)} \alpha_{\rho \rho_{5} \rho_{4}} \Delta_{\rho_{5}} \mathfrak{M}_{[i]}\left(-\boldsymbol{\delta}(\rho)-\boldsymbol{\delta}\left(\rho_{5}\right)\right)_{\boldsymbol{m}} .
\end{aligned}
$$

and, for $i=\mathfrak{l}+1$, we define

$$
\mathfrak{D}_{[\mathfrak{l}+1], \boldsymbol{m}}^{\rho}:=\sum_{\rho^{\prime} \in \Gamma_{[\mathfrak{l}+1]}(\rho)} \Delta_{\rho^{\prime}} \mathfrak{M}_{[\mathfrak{l}+1]}\left(-\boldsymbol{\delta}(\rho)-\boldsymbol{\delta}\left(\rho^{\prime}\right)\right)_{\boldsymbol{m}} .
$$

These are the contributions of $[\rho]$ toward the initial of $D_{[i]}, 1 \leq i \leq \mathfrak{l}+1$, corresponding to the generators identified in Equation (6.2), and the results from Lemmas 6.8 and 6.10. Then, we can use the above definition and Lemma 6.6 to provide an upper bound on the dimension of $H_{0}\left(\mathcal{I}_{[i]}\right)$ that improves upon the one presented in Proposition 6.7. 
Corollary 6.11 Given $\boldsymbol{m} \in \mathbb{Z}_{\geq 0}^{2}$ and $1 \leq i \leq \mathfrak{l}+1$, the following upper bound on the dimension of the $\boldsymbol{m}^{\text {th }}$ graded piece of $H_{0}\left(\mathcal{I}_{[i]}\right)$ holds:

$$
\operatorname{dim}\left(H_{0}\left(\mathcal{I}_{[i]}\right)\right)_{\boldsymbol{m}} \leq \sum_{\rho \in \operatorname{MS}_{[i]}} \operatorname{dim}\left(\mathfrak{M}_{[i]}(-\boldsymbol{\delta}(\rho))\right)_{\boldsymbol{m}}-\operatorname{dim}\left(\mathfrak{D}_{[i], \boldsymbol{m}}^{\rho}\right)
$$

\section{Configurations with stable dimension}

In this section, we outline sufficient conditions that guarantee that the dimension of the spline space $\mathcal{S}_{\boldsymbol{\Delta} \boldsymbol{m}, \boldsymbol{m}}$ can be computed exactly. We will work in a setting where Assumption 3 is true, i.e., where the bounds from Theorems 6.3 and 6.4 hold. Note that supplementary Macaulay2 scripts accompanying some of the examples presented in this section and the next can be downloaded from [34].

Theorem 7.1 Given $\boldsymbol{m} \in \mathbb{Z}_{\geq 0}^{2}$ and Assumptions 1-3, if $\operatorname{dim}\left(H_{0}\left(\mathcal{I}_{[i]}\right)\right)_{\boldsymbol{m}}=$ $\operatorname{dim}\left(H_{0}\left(\mathcal{C}_{[i]}\right)\right)_{\boldsymbol{m}}$ for all $1 \leq i \leq \mathfrak{l}+1$, then $h_{\Delta \boldsymbol{m}, \boldsymbol{m}}^{\boldsymbol{r}}=0$, i.e.,

$$
\operatorname{dim}\left(\mathcal{S}_{\Delta \boldsymbol{m}}^{\boldsymbol{r}}\right)_{\boldsymbol{m}}=\chi(\mathcal{Q})_{m}
$$

As per Theorem 7.1, if the upper bound in Corollary 6.11 equals $\operatorname{dim}\left(H_{0}\left(\mathcal{C}_{[i]}\right)\right)_{m}$ then the dimension of the spline space can be exactly determined. Before presenting a result that simplifies the computation of that upper bound, let us first consider an example where we explicitly use the results from the previous section to compute the spline space dimension.

Example 7.2 Consider the T-mesh shown in Fig. 4 and let $\boldsymbol{r}(\tau)=1$ for all interior edges. Let us consider two different degree deficit distributions on this mesh and find the dimension of the resulting spline space. In all of the following cases, the bismoothness for each maximal segment is simply $\delta(\rho)=(2,0)$ or $(0,2)$. We will also use the following fact that is implied by Proposition 3.12 for real numbers $a_{1} \neq a_{2}$ and $\boldsymbol{m} \geq(3,3)$ :

$$
\begin{aligned}
\operatorname{dim}\left(\mathfrak{M}_{[i]}(0,-2)\right)_{\boldsymbol{m}} & =\operatorname{dim}\left(\left(s+a_{1} u\right)^{2} \mathfrak{M}_{[i]}(-2,-2)+\left(s+a_{2} u\right)^{2} \mathfrak{M}_{[i]}(-2,-2)\right)_{\boldsymbol{m}}, \\
\operatorname{dim}\left(\mathfrak{M}_{[i]}(-2,0)\right)_{\boldsymbol{m}} & =\operatorname{dim}\left(\left(t+a_{1} v\right)^{2} \mathfrak{M}_{[i]}(-2,-2)+\left(t+a_{2} v\right)^{2} \mathfrak{M}_{[i]}(-2,-2)\right)_{\boldsymbol{m}} .(\star)
\end{aligned}
$$

(a) Let $\boldsymbol{\Delta} \boldsymbol{m}(\sigma)=(1,1)$ for all faces $\sigma \in \mathcal{T}$ except for the face bounded by vertices $\gamma_{4}, \gamma_{8}, \gamma_{12}, \gamma_{11}$; on the latter face, the degree deficit is chosen to be $(0,0)$. Let us choose the associated degree-deficit sequence as $\Delta \boldsymbol{m}_{0}=(0,0)<\Delta \boldsymbol{m}_{1}=$ $(1,1)$ so that $\mathfrak{l}=1$. We will choose $\boldsymbol{m}=(3,3)$. The following results follow on the different active meshes $\mathcal{T}_{[i]}, 1 \leq i \leq \mathfrak{l}+1=2$. 


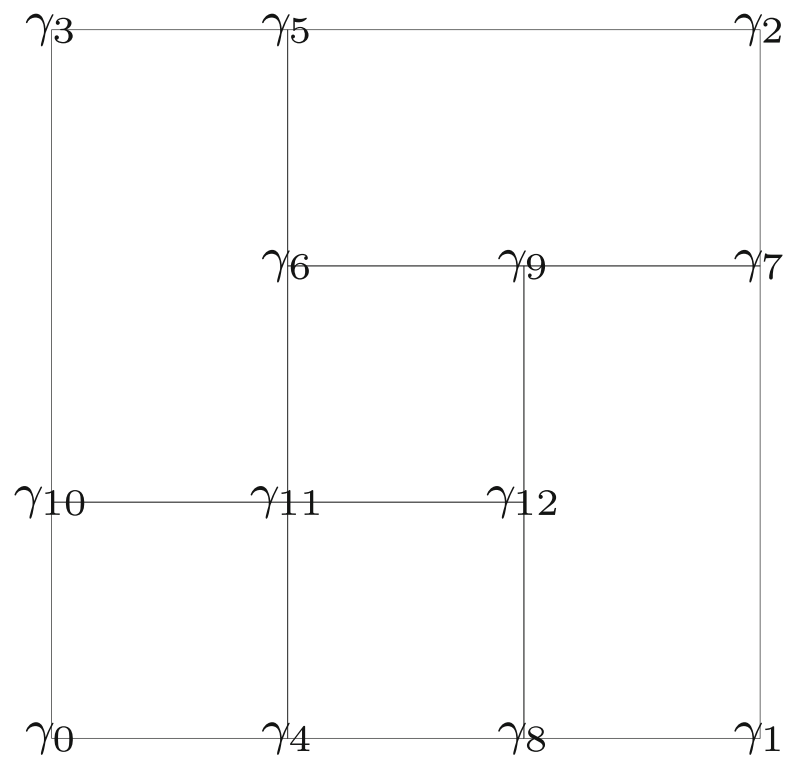

Fig. 4 The T-mesh used in Example 7.2 to explicitly demonstrate how results from Section 3 can be used to find the dimension of $\mathcal{S}_{\Delta m, m}^{r}$

$-[i=2]$ : From Proposition 4.5, $\operatorname{dim}\left(H_{0}\left(\mathcal{C}_{[i]}\right)\right)_{m}=0$. Furthermore, from Proposition 5.7, there are no interior maximal segments therefore $\operatorname{dim}\left(H_{0}\left(\mathcal{I}_{[i]}\right)\right)_{m}=0$.

- $\quad[i=1]$ : From Proposition 4.5, $\operatorname{dim}\left(H_{0}\left(\mathcal{C}_{[i]}\right)\right)_{m}=0$. Furthermore, there is a single active interior maximal segment $\rho=\gamma_{11} \gamma_{12}$, and the non-interior maximal segments $\rho_{1}=\gamma_{4} \gamma_{11}$ and $\rho_{2}=\gamma_{8} \gamma_{12}$ intersect it. Therefore, $\rho_{1}, \rho_{2} \in \Gamma_{[i]}(\rho)$ and from Proposition 6.7 and Equation $(\star)$ above, we get

$$
\operatorname{dim}\left(H_{0}\left(\mathcal{I}_{[i]}\right)\right)_{\boldsymbol{m}} \leq \operatorname{dim}\left(\mathfrak{M}_{[i]}(0,-2)\right)_{\boldsymbol{m}}-\operatorname{dim}\left(\sum_{\rho^{\prime} \in\left\{\rho_{1}, \rho_{2}\right\}} \Delta_{\rho^{\prime}} \mathfrak{M}_{[i]}(-2,-2)\right)_{\boldsymbol{m}}=0 .
$$

From the above, we can see that Theorem 7.1 applies. Thus, the dimension of $\mathcal{S}_{\boldsymbol{\Delta} \boldsymbol{m}, \boldsymbol{m}}^{r}$ can be determined to be exactly 17. It can be observed using the Macaulay2 script [34, ex0a.m2] that $\boldsymbol{m}=(3,3)$ is also the smallest degree for which we get an increase in the dimension because of the non-uniformity in polynomial degrees.

(b) Let $\boldsymbol{\Delta} \boldsymbol{m}(\sigma)=(1,1)$ for all faces $\sigma \in \mathcal{T}$ except for the face bounded by vertices $\gamma_{6}, \gamma_{9}, \gamma_{12}, \gamma_{11}$; on the latter face, the degree deficit is chosen to be $(0,0)$. Let us choose the associated degree-deficit sequence as $\boldsymbol{\Delta} \boldsymbol{m}_{0}=(0,0)<\boldsymbol{\Delta} \boldsymbol{m}_{1}=$ $(1,1)$ so that $\mathfrak{l}=1$. We will choose $\boldsymbol{m}=(4,4)$. The following results follow on the different active meshes $\mathcal{T}_{[i]}, 1 \leq i \leq \mathfrak{l}+1=2$. 
$-\quad[i=2]$ : From Proposition 4.5, $\operatorname{dim}\left(H_{0}\left(\mathcal{C}_{[i]}\right)\right)_{\boldsymbol{m}}=0$. Furthermore, from Proposition 5.7, there are no interior maximal segments therefore $\operatorname{dim}\left(H_{0}\left(\mathcal{I}_{[i]}\right)\right)_{\boldsymbol{m}}=0$.

- $\quad[i=1]$ : From Proposition 4.5, $\operatorname{dim}\left(H_{0}\left(\mathcal{C}_{[i]}\right)\right)_{m}=9$. In this case, there are 4 active maximal segments $\rho_{1}=\gamma_{11} \gamma_{12}, \rho_{2}=\gamma_{12} \gamma_{9}, \rho_{3}=\gamma_{9} \gamma_{6}$, $\rho_{1}=\gamma_{6} \gamma_{11}$. Let us order these maximal segments as $\rho_{3}>\rho_{2}>\rho_{4}>\rho_{1}$. Therefore,

$$
\Gamma_{[i]}\left(\rho_{1}\right)=\emptyset, \quad \Gamma_{[i]}\left(\rho_{2}\right)=\Gamma_{[i]}\left(\rho_{4}\right)=\left\{\rho_{1}\right\}, \quad \Gamma_{[i]}\left(\rho_{3}\right)=\left\{\rho_{2}, \rho_{4}\right\} .
$$

Once again, from Proposition 6.7 and Equation $(\star)$, there is no contribution to $\operatorname{dim}\left(H_{0}\left(\mathcal{I}_{[i]}\right)\right)_{\boldsymbol{m}}$ from $\rho_{3}$. Furthermore, from Lemma 6.8 and Corollary 6.9, we can also verify that $\boldsymbol{\Delta} \boldsymbol{p}_{\rho_{3}}=(1,0)$ and $\Upsilon_{[i]}\left(\rho_{3}, \boldsymbol{m}\right)=$ $\left\{\left\{\left(\rho_{1}, \rho_{2}\right),\left(\rho_{1}, \rho_{4}\right)\right\}\right\}$. Therefore, from Lemma 6.10, we can state that:

$$
\left[\rho_{2}\right] \Delta_{\rho_{3}} \mathfrak{M}_{[i]}(-2,-2)_{m} \subset \text { In } D_{[i]}
$$

However, since $\rho_{1} \in \Gamma_{[i]}\left(\rho_{2}\right)$, we also have the containment

$$
\left[\rho_{2}\right] \Delta_{\rho_{1}} \mathfrak{M}_{[i]}(-2,-2)_{m} \subset \text { In } D_{[i]}
$$

Therefore, again from Equation $(\star)$, there is no contribution to $\operatorname{dim}\left(H_{0}\left(\mathcal{I}_{[i]}\right)\right)_{m}$ from $\rho_{2}$. Thus, the only contributions to the upper bound in Proposition 6.7 come from $\rho_{1}$ and $\rho_{4}$,

$$
\begin{aligned}
\operatorname{dim}\left(H_{0}\left(\mathcal{I}_{[i]}\right)\right)_{\boldsymbol{m}} \leq & \operatorname{dim}\left(\left[\rho_{1}\right] \mathfrak{M}_{[i]}(0,-2)\right)_{\boldsymbol{m}}+\operatorname{dim}\left(\left[\rho_{4}\right] \mathfrak{M}_{[i]}(-2,0)\right)_{\boldsymbol{m}} \\
& -\operatorname{dim}\left(\left[\rho_{4}\right] \Delta_{\rho_{1}} \mathfrak{M}_{[i]}(-2,-2)\right)_{\boldsymbol{m}} \\
= & 7+7-5=9=\operatorname{dim}\left(H_{0}\left(\mathcal{C}_{[i]}\right)\right)_{\boldsymbol{m}}
\end{aligned}
$$

Thus, $\operatorname{dim}\left(H_{0}\left(\mathcal{I}_{[i]}\right)\right)_{\boldsymbol{m}}=\operatorname{dim}\left(H_{0}\left(\mathcal{C}_{[i]}\right)\right)_{\boldsymbol{m}}$.

From the above, we can see that Theorem 7.1 applies. Thus, the dimension of $\mathcal{S}_{\boldsymbol{\Delta} \boldsymbol{m}, \boldsymbol{m}}^{\boldsymbol{r}}$ can be found to be 41 . As in part (a), it can be observed using the Macaulay2 script [34, ex0b.m2] that $\boldsymbol{m}=(4,4)$ is again the smallest degree for which we get an increase in the dimension because of the non-uniformity in polynomial degrees.

Let us now present a result that simplifies the computation of the upper bound on $\operatorname{dim}\left(H_{0}\left(\mathcal{I}_{[i]}\right)\right)$. Following Equations (6.6) and (6.7), let us define the sets:

$$
\begin{aligned}
\Lambda_{[i]}(\rho, \boldsymbol{m}): & =\left\{\rho^{\prime}: \rho^{\prime} \in \Gamma_{[i]}(\rho) \text {, or }(\cdot, \rho) \in \Theta_{[i]}\left(\rho^{\prime}, \boldsymbol{m}\right)\right. \text {, or } \\
& \left.\left(\rho, \rho^{\prime \prime}\right) \in \Theta_{[i]}\left(\rho^{\prime}, \boldsymbol{m}\right) \text { and } \alpha_{\rho \rho^{\prime} \rho^{\prime \prime}}=1\right\}, \quad 1 \leq i \leq \mathfrak{l}, \\
\Lambda_{[\mathfrak{l}+1]}(\rho, \boldsymbol{m}): & =\Gamma_{[\mathfrak{l}+1]}(\rho) .
\end{aligned}
$$


Definition 7.3 (Maximal-segment weights) For $\rho \in \operatorname{MS}_{[i]}, \boldsymbol{m}=\left(m, m^{\prime}\right) \in \mathbb{Z}_{\geq 0}^{2}$ and $1 \leq i \leq \mathfrak{l}+1$, the weight of $\rho$ is denoted by $\omega_{[i], \boldsymbol{m}}^{\rho}$, and it is defined to be

$$
\omega_{[i], \boldsymbol{m}}^{\rho}:= \begin{cases}\sum_{\rho^{\prime} \in \Lambda_{[i]}(\rho, \boldsymbol{m})}\left(m-\Delta m_{(i-1) 1}-\boldsymbol{r}\left(\rho^{\prime}\right)\right)_{+}, & \rho \in{ }^{h} \mathrm{MS}_{[i]}, \\ \sum_{\rho^{\prime} \in \Lambda_{[i]}(\rho, \boldsymbol{m})}\left(m^{\prime}-\Delta m_{(i-1) 2}-\boldsymbol{r}\left(\rho^{\prime}\right)\right)_{+}, & \rho \in{ }^{v} \mathrm{MS}_{[i]} .\end{cases}
$$

Lemma 7.4 For $\boldsymbol{m}=\left(m, m^{\prime}\right) \in \mathbb{Z}_{\geq 0}^{2}$ and $1 \leq i \leq \mathfrak{l}+1$,

$$
\omega_{[i], \boldsymbol{m}}^{\rho} \geq\left\{\begin{array}{ll}
m-\Delta m_{(i-1) 1}+1, & \rho \in{ }^{h} \mathrm{MS}_{[i]} \\
m^{\prime}-\Delta m_{(i-1) 2}+1, & \rho \in{ }^{v} \mathrm{MS}_{[i]}
\end{array} \Rightarrow \mathfrak{D}_{[i], \boldsymbol{m}}^{\rho}=\mathfrak{M}_{[i]}(-\boldsymbol{\delta}(\rho))_{\boldsymbol{m}} .\right.
$$

In particular, if $\omega_{[i], m}^{\rho}$ satisfies the above sufficient condition, then $\rho$ will not contribute to the dimension of $\operatorname{dim}\left(H_{0}\left(\mathcal{I}_{[i]}\right)\right)$.

Proof The claimed implication follows from Proposition 3.12 and, subsequently, Corollary 6.11 implies that $\rho$ does not contribute to $\operatorname{dim}\left(H_{0}\left(\mathcal{I}_{[i]}\right)\right)$.

Example 7.5 Let us revisit Example 7.2(b) and see how Lemma 7.4 and the notion of maximal-segment weights simplifies the computation of (bounds on) the spline space dimension. For $i=2$ there are no interior maximal segments; so, let us look at the case of $i=1$. We have

$$
\Gamma_{[i]}\left(\rho_{3}\right)=\left\{\rho_{2}, \rho_{4}\right\}, \quad \Gamma_{[i]}\left(\rho_{2}\right)=\left\{\rho_{1}\right\}, \quad \Gamma_{[i]}\left(\rho_{4}\right)=\left\{\rho_{1}\right\}, \quad \Theta_{[i]}\left(\rho_{3}, \boldsymbol{m}\right)=\left\{\left(\rho_{2}, \rho_{4}\right)\right\} .
$$

Thus, from Equation (7.1), we have:

$\Lambda_{[i]}\left(\rho_{1}, \boldsymbol{m}\right)=\emptyset, \quad \Lambda_{[i]}\left(\rho_{2}, \boldsymbol{m}\right)=\left\{\rho_{1}, \rho_{3}\right\}, \quad \Lambda_{[i]}\left(\rho_{3}, \boldsymbol{m}\right)=\left\{\rho_{2}, \rho_{4}\right\}, \quad \Lambda_{[i]}\left(\rho_{4}, \boldsymbol{m}\right)=\left\{\rho_{1}\right\}$,

and from Definition 7.3, for $\rho \in\left\{\rho_{2}, \rho_{3}\right\}$ we obtain:

$$
\omega_{[i], \boldsymbol{m}}^{\rho}=2 \times(4-1-1)=4 .
$$

Then, from Lemma 7.4, we see that $\rho_{2}$ and $\rho_{3}$ do not contribute to $\operatorname{dim}\left(H_{0}\left(\mathcal{I}_{[i]}\right)\right)_{\boldsymbol{m}}$.

\section{Examples}

In this section, we provide three examples that illustrate how the theory developed in this document can be used to compute the spline space dimension in the presence of non-uniform degrees. The first two examples show configurations where Theorem 7.1 applies, i.e., where the dimension can be computed exactly. The last example serves to counter the expectation that Theorem 7.1 will apply in all circumstances. 
Remark 8.1 This article is focused on the development of theoretical tools that help compute or estimate the dimension of non-uniform degree spline spaces. Thus, our aim in this section is to present explicit examples that illustrate the utility of our results in this context. Presenting applications of such spline spaces to finite element computations, for instance, is beyond the scope of this paper and involves several open research questions such as the computation of a normalized, well-conditioned basis for the spline spaces. This is a well-understood problem in the univariate setting (e.g., [30, 37]), but is a topic of ongoing research in the multivariate setting (e.g., $[21,33])$. This article can thus be viewed as a theoretical companion to any (existing and future) application oriented efforts. For instance, when studying a spline space, dimension counts can help certify if a linearly independent set of splines in it forms a basis for it.

Example 8.2 Consider the T-mesh shown in Fig. 5. Let us build a $C^{1}$ spline space on this mesh, i.e., $\boldsymbol{r}(\tau)=1$ for all interior edges $\tau$. The degree deficit on the shaded faces is chosen to be $(0,0)$ and on the white faces it is chosen to be $(1,1)$. We choose $\Delta \boldsymbol{m}_{0}=(0,0)$ and $\boldsymbol{\Delta} \boldsymbol{m}_{1}=(1,1)$, i.e., $\mathfrak{l}=1$, and choose $\boldsymbol{m}=(3,3)$. Let us examine the active T-meshes $\mathcal{T}_{[i]}$ in the following for $i=1,2$.

- $\quad[i=2]:$ The only interior maximal segments in $\mathcal{T}_{[i]}$ are

$$
\rho_{1}=\gamma_{20} \gamma_{22}, \rho_{2}=\gamma_{23} \gamma_{25}, \rho_{3}=\gamma_{26} \gamma_{28}, \rho_{4}=\gamma_{29} \gamma_{31} .
$$

Let us order these interior maximal segments as $\rho_{1}<\rho_{2}<\rho_{3}<\rho_{4}$. Then, notice that the cardinality of $\Gamma_{[i]}\left(\rho_{j}\right)$ is 3 for each $\rho_{j}$. Therefore, we can compute the weight of each interior maximal segment to be $\omega_{[i], m}^{\rho_{j}} \geq 3$ for all $j$. Then, using Lemma 7.4, we see that

$$
\operatorname{dim}\left(H_{0}\left(\mathcal{I}_{[i]}\right)\right)_{\boldsymbol{m}}=\operatorname{dim}\left(H_{0}\left(\mathcal{C}_{[i]}\right)\right)_{\boldsymbol{m}}=0
$$

Fig. 5 A non-uniform degree $C^{1}$ spline space consisting of quadratic and cubic polynomial pieces is built on the above mesh. Example 8.2 shows that the dimension of the spline space can be computed using Theorem 7.1

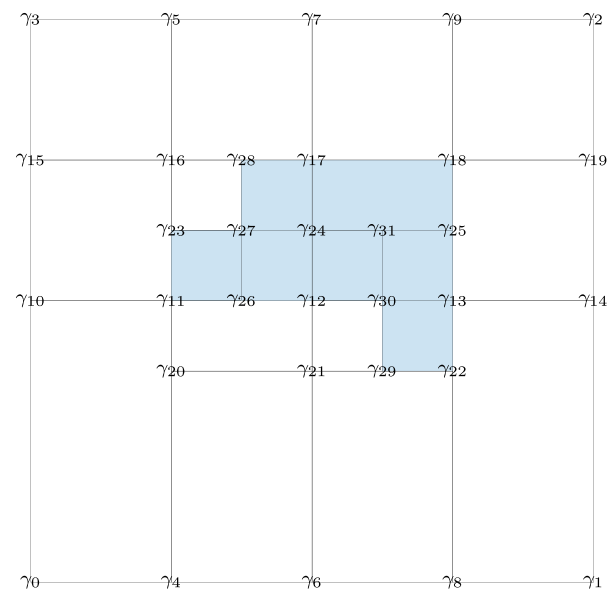


$-\quad[i=1]$ : All maximal segments in $\mathcal{T}_{[i]}$ are interior maximal segments, let us denote them as below:

$$
\begin{aligned}
& \rho_{1}=\gamma_{11} \gamma_{13}, \rho_{2}=\gamma_{22} \gamma_{18}, \rho_{3}=\gamma_{29} \gamma_{31}, \rho_{4}=\gamma_{12} \gamma_{17}, \rho_{5}=\gamma_{26} \gamma_{28}, \\
& \rho_{6}=\gamma_{11} \gamma_{23}, \rho_{7}=\gamma_{29} \gamma_{22}, \rho_{8}=\gamma_{23} \gamma_{25}, \rho_{9}=\gamma_{28} \gamma_{18} .
\end{aligned}
$$

Let us order these interior maximal segments so that $\rho_{j}<\rho_{k}$ when $j<k$. The sets $\Gamma_{[i]}\left(\rho_{j}\right)$ can be seen to be

$$
\begin{aligned}
& \Gamma_{[i]}\left(\rho_{1}\right)=\emptyset, \Gamma_{[i]}\left(\rho_{2}\right)=\Gamma_{[i]}\left(\rho_{3}\right)=\Gamma_{[i]}\left(\rho_{4}\right)=\Gamma_{[i]}\left(\rho_{5}\right)=\Gamma_{[i]}\left(\rho_{6}\right)=\left\{\rho_{1}\right\}, \\
& \Gamma_{[i]}\left(\rho_{7}\right)=\left\{\rho_{2}, \rho_{3}\right\}, \Gamma_{[i]}\left(\rho_{8}\right)=\left\{\rho_{2}, \rho_{3}, \rho_{4}, \rho_{5}, \rho_{6}\right\}, \Gamma_{[i]}\left(\rho_{9}\right)=\left\{\rho_{2}, \rho_{4}, \rho_{5}\right\} .
\end{aligned}
$$

Next, it can be seen from Corollary 6.9 that

$$
\Upsilon_{[i]}\left(\rho_{8}, \boldsymbol{m}\right) \ni \Upsilon:=\left\{\left(\rho_{1}, \rho_{2}\right),\left(\rho_{1}, \rho_{3}\right),\left(\rho_{1}, \rho_{4}\right),\left(\rho_{1}, \rho_{5}\right),\left(\rho_{1}, \rho_{6}\right)\right\} .
$$

Then, from Lemma 6.10, we can build the sets $\Lambda_{[i]}\left(\rho_{j}\right)$ for $j=3, \ldots, 6$ such that

$$
\left\{\rho_{1}, \rho_{8}\right\} \subseteq \Lambda_{[i]}\left(\rho_{3}\right), \Lambda_{[i]}\left(\rho_{4}\right), \Lambda_{[i]}\left(\rho_{5}\right), \Lambda_{[i]}\left(\rho_{6}\right) .
$$

Then, we see that the weight $\omega_{[i], m}^{\rho_{j}} \geq 4$ for all $j=3, \ldots, 9$, and thus the upper bound on the dimension of $H_{0}\left(\mathcal{I}_{[i]}\right)$ can be computed to be the following:

$$
\begin{aligned}
\operatorname{dim}\left(H_{0}\left(\mathcal{I}_{[i]}\right)\right)_{\boldsymbol{m}} \leq & \operatorname{dim}\left(\left[\rho_{1}\right] \mathfrak{M}_{[i]}(0,-2)\right)_{\boldsymbol{m}}+\operatorname{dim}\left(\left[\rho_{2}\right] \mathfrak{M}_{[i]}(-2,0)\right)_{\boldsymbol{m}} \\
& -\operatorname{dim}\left(\left[\rho_{2}\right] \Delta_{\rho_{1}} \mathfrak{M}_{[i]}(-2,-2)\right)_{\boldsymbol{m}} \\
= & 5+5-3=7=\operatorname{dim}\left(H_{0}\left(\mathcal{C}_{[i]}\right)\right)_{\boldsymbol{m}} .
\end{aligned}
$$

Therefore, $\operatorname{dim}\left(H_{0}\left(\mathcal{I}_{[i]}\right)\right)_{\boldsymbol{m}}=\operatorname{dim}\left(H_{0}\left(\mathcal{C}_{[i]}\right)\right)_{\boldsymbol{m}}$.

From the above, we can see that Theorem 7.1 applies and

$$
\operatorname{dim}\left(\mathcal{S}_{\boldsymbol{\Delta} \boldsymbol{m}}^{\boldsymbol{r}}\right)_{\boldsymbol{m}}=\chi(\mathcal{Q})_{\boldsymbol{m}}=37 .
$$

In particular, there are 30 splines on $\mathcal{T}_{[2]}$ and 7 on $\mathcal{T}_{[1]}$. The reader can use the accompanying Macaulay2 script [34, ex1.m2] to confirm that $\boldsymbol{m}=(3,3)$ is the smallest bi-degree for which non-uniformity in degrees leads to an increase in the dimension.

Example 8.3 Consider the T-mesh shown in Fig. 6. Let us build a $C^{2}$ spline space on this mesh, i.e., $\boldsymbol{r}(\tau)=2$ for all interior edges $\tau$. The degree deficit on the shaded faces is chosen to be $(0,0)$ and on the white faces it is chosen to be $(1,1)$. We choose $\Delta \boldsymbol{m}_{0}=(0,0)$ and $\boldsymbol{\Delta} \boldsymbol{m}_{1}=(1,1)$, i.e., $\mathfrak{l}=1$, and choose $\boldsymbol{m}=(4,4)$. Let us examine the active T-meshes $\mathcal{T}_{[i]}$ in the following for $i=1,2$.

- $\quad[i=2]:$ The only interior maximal segments in $\mathcal{T}_{[i]}$ are

$$
\rho_{1}=\gamma_{35} \gamma_{39}, \rho_{2}=\gamma_{40} \gamma_{44}, \rho_{3}=\gamma_{45} \gamma_{49} .
$$

We can order them in any manner with respect to each other since they do not intersect each other. The weight of each interior maximal segment can be computed to be $\omega_{[i], m}^{\rho_{j}}=5$ for all $j$. Then, using Lemma 7.4, we see that

$$
\operatorname{dim}\left(H_{0}\left(\mathcal{I}_{[i]}\right)\right)_{\boldsymbol{m}}=\operatorname{dim}\left(H_{0}\left(\mathcal{C}_{[i]}\right)\right)_{\boldsymbol{m}}=0 .
$$




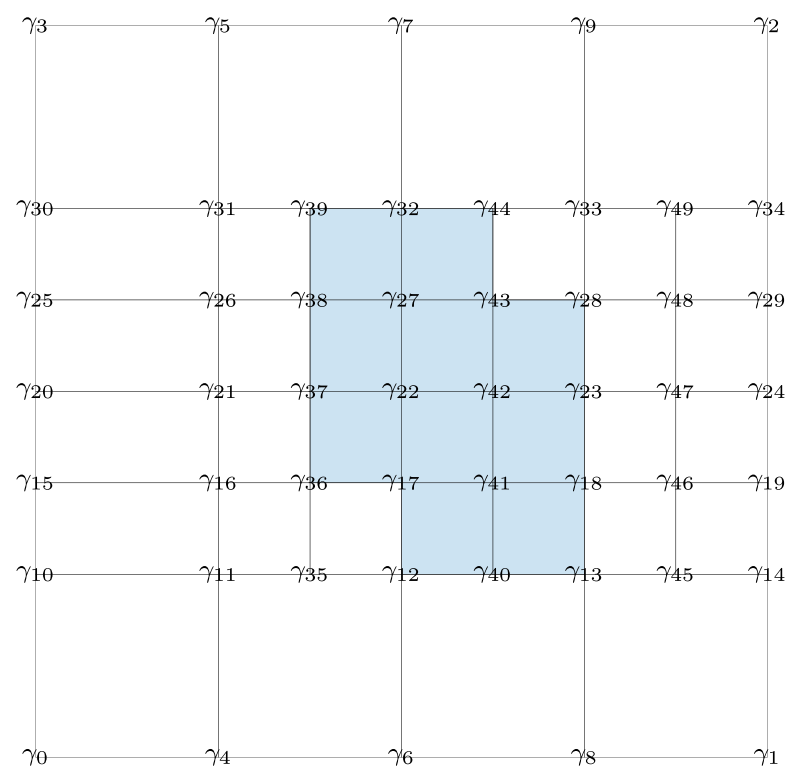

Fig. 6 A non-uniform degree $C^{2}$ spline space consisting of cubic and quartic polynomial pieces is built on the above mesh. Example 8.3 shows that the dimension of the spline space can be computed using Theorem 7.1

- $\quad[i=1]$ : All maximal segments in $\mathcal{T}_{[i]}$ are interior maximal segments, let us denote them as below:

$$
\begin{aligned}
& \rho_{1}=\gamma_{12} \gamma_{32}, \rho_{2}=\gamma_{36} \gamma_{18}, \rho_{3}=\gamma_{12} \gamma_{13}, \rho_{4}=\gamma_{37} \gamma_{23}, \rho_{5}=\gamma_{38} \gamma_{28}, \\
& \rho_{6}=\gamma_{39} \gamma_{44}, \rho_{7}=\gamma_{36} \gamma_{39}, \rho_{8}=\gamma_{40} \gamma_{44}, \rho_{9}=\gamma_{13} \gamma_{28} .
\end{aligned}
$$

Let us order these interior maximal segments so that $\rho_{j}<\rho_{k}$ when $j<k$. The sets $\Gamma_{[i]}\left(\rho_{j}\right)$ can be seen to be:

$$
\begin{aligned}
& \Gamma_{[i]}\left(\rho_{1}\right)=\emptyset, \Gamma_{[i]}\left(\rho_{2}\right)=\Gamma_{[i]}\left(\rho_{3}\right)=\Gamma_{[i]}\left(\rho_{4}\right)=\Gamma_{[i]}\left(\rho_{5}\right)=\Gamma_{[i]}\left(\rho_{6}\right)=\left\{\rho_{1}\right\} \\
& \Gamma_{[i]}\left(\rho_{7}\right)=\left\{\rho_{2}, \rho_{4}, \rho_{5}, \rho_{6}\right\}, \Gamma_{[i]}\left(\rho_{8}\right)=\left\{\rho_{2}, \rho_{3}, \rho_{4}, \rho_{5}, \rho_{6}\right\}, \Gamma_{[i]}\left(\rho_{9}\right)=\left\{\rho_{2}, \rho_{3}, \rho_{4}, \rho_{5}\right\}
\end{aligned}
$$

Next, it can be seen from Corollary 6.9 that

$$
\begin{aligned}
& \Upsilon_{[i]}\left(\rho_{7}, \boldsymbol{m}\right) \ni\left\{\left(\rho_{1}, \rho_{2}\right),\left(\rho_{1}, \rho_{4}\right),\left(\rho_{1}, \rho_{5}\right),\left(\rho_{1}, \rho_{6}\right)\right\}, \\
& \Upsilon_{[i]}\left(\rho_{8}, \boldsymbol{m}\right) \ni\left\{\left(\rho_{1}, \rho_{2}\right),\left(\rho_{1}, \rho_{3}\right),\left(\rho_{1}, \rho_{4}\right),\left(\rho_{1}, \rho_{5}\right),\left(\rho_{1}, \rho_{6}\right)\right\}, \\
& \Upsilon_{[i]}\left(\rho_{9}, \boldsymbol{m}\right) \ni\left\{\left(\rho_{1}, \rho_{2}\right),\left(\rho_{1}, \rho_{3}\right),\left(\rho_{1}, \rho_{4}\right),\left(\rho_{1}, \rho_{5}\right)\right\} .
\end{aligned}
$$

Then, from Lemma 6.10, we can build the sets $\Lambda_{[i]}\left(\rho_{j}\right)$ for $j=3, \ldots, 6$ such that

$$
\left\{\rho_{1}, \rho_{8}, \rho_{9}\right\} \subseteq \Lambda_{[i]}\left(\rho_{3}\right),\left\{\rho_{1}, \rho_{7}, \rho_{8}\right\} \subseteq \Lambda_{[i]}\left(\rho_{4}\right), \Lambda_{[i]}\left(\rho_{5}\right), \Lambda_{[i]}\left(\rho_{6}\right)
$$




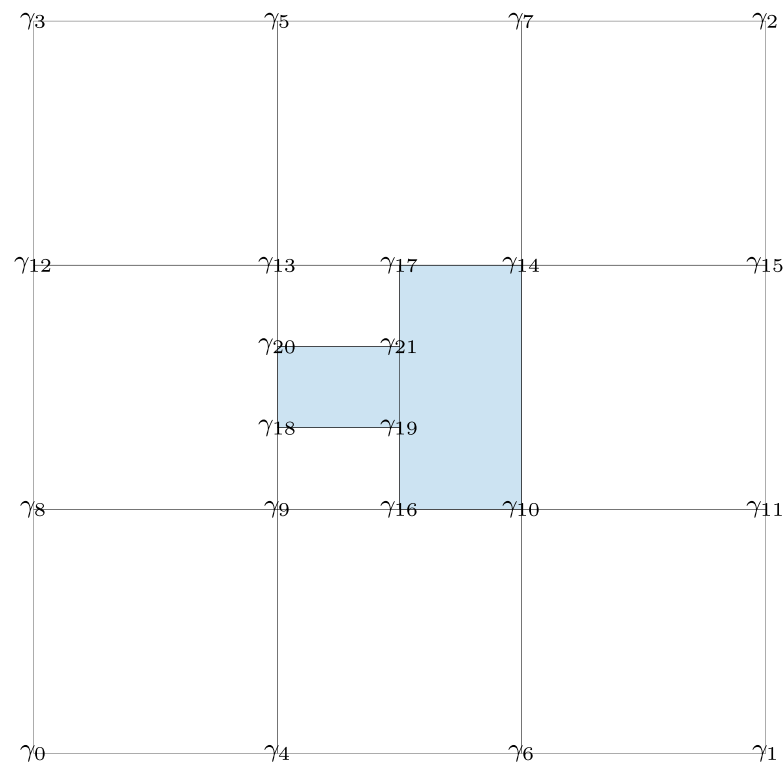

Fig. 7 A non-uniform degree $C^{2}$ spline space consisting of cubic and quartic polynomial pieces is built on the above mesh. Example 8.4 shows that the dimension of the spline space coincides with the upper bound implied by Theorem 7.1

Then, we see that the weight $\omega_{[i], m}^{\rho_{j}}>5$ for all $j=3, \ldots, 9$, and thus the upper bound on the dimension of $H_{0}\left(\mathcal{I}_{[i]}\right)$ can be computed to be the following:

$$
\begin{aligned}
\operatorname{dim}\left(H_{0}\left(\mathcal{I}_{[i]}\right)\right)_{\boldsymbol{m}} \leq & \operatorname{dim}\left(\left[\rho_{1}\right] \mathfrak{M}_{[i]}(0,-3)\right)_{\boldsymbol{m}}+\operatorname{dim}\left(\left[\rho_{2}\right] \mathfrak{M}_{[i]}(-3,0)\right)_{\boldsymbol{m}} \\
& -\operatorname{dim}\left(\left[\rho_{2}\right] \Delta_{\rho_{1}} \mathfrak{M}_{[i]}(-3,-3)\right)_{\boldsymbol{m}} \\
= & 6+6-3=9=\operatorname{dim}\left(H_{0}\left(\mathcal{C}_{[i]}\right)\right)_{\boldsymbol{m}} .
\end{aligned}
$$

Therefore, $\operatorname{dim}\left(H_{0}\left(\mathcal{I}_{[i]}\right)\right)_{\boldsymbol{m}}=\operatorname{dim}\left(H_{0}\left(\mathcal{C}_{[i]}\right)\right)_{\boldsymbol{m}}$.

From the above, we can see that Theorem 7.1 applies and

$$
\operatorname{dim}\left(\mathcal{S}_{\Delta m}^{r}\right)_{m}=\chi(\mathcal{Q})_{m}=75
$$

In particular, there are 66 splines on $\mathcal{T}_{[2]}$ and 9 on $\mathcal{T}_{[1]}$. The reader can use the accompanying Macaulay2 script [34, ex2.m2] to confirm that $\boldsymbol{m}=(4,4)$ is the smallest bi-degree for which non-uniformity in degrees leads to an increase in the dimension.

Example 8.4 Consider the T-mesh shown in Fig. 7. Let us build a $C^{2}$ spline space on this mesh, i.e., $\boldsymbol{r}(\tau)=2$ for all interior edges $\tau$. The degree deficit on the shaded faces is chosen to be $(0,0)$ and on the white faces it is chosen to be $(1,1)$. We choose $\Delta \boldsymbol{m}_{0}=(0,0)$ and $\boldsymbol{\Delta} \boldsymbol{m}_{1}=(1,1)$, i.e., $\mathfrak{l}=1$, and choose $\boldsymbol{m}=(6,6)$. Let us examine the active T-meshes $\mathcal{T}_{[i]}$ in the following for $i=1,2$. 
$-\quad[i=2]:$ The only interior maximal segments in $\mathcal{T}_{[i]}$ are

$$
\rho_{1}=\gamma_{16} \gamma_{17}, \rho_{2}=\gamma_{20} \gamma_{21}, \rho_{3}=\gamma_{18} \gamma_{19} .
$$

Let us order them as $\rho_{1}<\rho_{2}<\rho_{3}$. Then, for $\boldsymbol{m}=(6,6)$, it can be computed that $\omega_{[i], m}^{\rho_{j}} \geq 6$. Then, using Lemma 7.4, we see that

$$
\operatorname{dim}\left(H_{0}\left(\mathcal{I}_{[i]}\right)\right)_{\boldsymbol{m}}=\operatorname{dim}\left(H_{0}\left(\mathcal{C}_{[i]}\right)\right)_{\boldsymbol{m}}=0 .
$$

Note that $\boldsymbol{m}=(6,6)$ is the smallest bi-degree in which $H_{0}\left(\mathcal{I}_{[i]}\right)$ vanishes.

- $\quad[i=1]$ : All maximal segments in $\mathcal{T}_{[i]}$ are interior maximal segments, let us denote them as below:

$$
\begin{aligned}
& \rho_{1}=\gamma_{16} \gamma_{17}, \rho_{2}=\gamma_{16} \gamma_{10}, \rho_{3}=\gamma_{17} \gamma_{14}, \rho_{4}=\gamma_{10} \gamma_{14}, \\
& \rho_{5}=\gamma_{18} \gamma_{19}, \rho_{6}=\gamma_{20} \gamma_{21}, \rho_{7}=\gamma_{18} \gamma_{20} .
\end{aligned}
$$

Let us order these interior maximal segments so that $\rho_{j}<\rho_{k}$ when $j<k$. The sets $\Gamma_{[i]}\left(\rho_{j}\right)$ can be seen to be:

$$
\begin{aligned}
& \Gamma_{[i]}\left(\rho_{1}\right)=\emptyset, \Gamma_{[i]}\left(\rho_{2}\right)=\Gamma_{[i]}\left(\rho_{3}\right)=\Gamma_{[i]}\left(\rho_{5}\right)=\Gamma_{[i]}\left(\rho_{6}\right)=\left\{\rho_{1}\right\}, \\
& \Gamma_{[i]}\left(\rho_{4}\right)=\left\{\rho_{2}, \rho_{3}\right\}, \Gamma_{[i]}\left(\rho_{7}\right)=\left\{\rho_{5}, \rho_{6}\right\} .
\end{aligned}
$$

Next, it can be seen from Corollary 6.9 that

$$
\begin{aligned}
& \Upsilon_{[i]}\left(\rho_{4}, \boldsymbol{m}\right) \ni\left\{\left(\rho_{1}, \rho_{2}\right),\left(\rho_{1}, \rho_{3}\right)\right\}, \\
& \Upsilon_{[i]}\left(\rho_{7}, \boldsymbol{m}\right) \ni\left\{\left(\rho_{1}, \rho_{5}\right),\left(\rho_{1}, \rho_{6}\right)\right\} .
\end{aligned}
$$

Then, from Lemma 6.10, we can build the sets $\Lambda_{[i]}\left(\rho_{3}\right)$ and $\Lambda_{[i]}\left(\rho_{6}\right)$ such that

$$
\Lambda_{[i]}\left(\rho_{3}\right)=\left\{\rho_{1}, \rho_{4}\right\}, \Lambda_{[i]}\left(\rho_{6}\right)=\left\{\rho_{1}, \rho_{7}\right\} .
$$

Then, we see that the weight $\omega_{[i], m}^{\rho_{j}} \geq 8$ for $j=3,4,6,7$. Thus, the upper bound on the dimension of $H_{0}\left(\mathcal{I}_{[i]}\right)$ can be computed to be the following:

$$
\begin{aligned}
\operatorname{dim}\left(H_{0}\left(\mathcal{I}_{[i]}\right)\right)_{\boldsymbol{m}} \leq \operatorname{dim} & \left(\left[\rho_{1}\right] \mathfrak{M}_{[i]}(0,-3)\right)_{\boldsymbol{m}}+\operatorname{dim}\left(\left[\rho_{2}\right] \mathfrak{M}_{[i]}(-3,0)\right)_{\boldsymbol{m}} \\
& +\operatorname{dim}\left(\left[\rho_{5}\right] \mathfrak{M}_{[i]}(0,-3)\right)_{\boldsymbol{m}}-\operatorname{dim}\left(\left[\rho_{2}\right] \Delta_{\rho_{1}} \mathfrak{M}_{[i]}(-3,-3)\right)_{\boldsymbol{m}} \\
& -\operatorname{dim}\left(\left[\rho_{5}\right] \Delta_{\rho_{1}} \mathfrak{M}_{[i]}(-3,-3)\right)_{\boldsymbol{m}} \\
=10 & +10+10-7-7=16 .
\end{aligned}
$$

On the other hand, using Proposition 4.5, we can compute that

$$
\operatorname{dim}\left(H_{0}\left(\mathcal{C}_{[i]}\right)\right)_{m}=13 .
$$

Therefore, $\operatorname{dim}\left(H_{0}\left(\mathcal{I}_{[i]}\right)\right)_{\boldsymbol{m}}-\operatorname{dim}\left(H_{0}\left(\mathcal{C}_{[i]}\right)\right)_{\boldsymbol{m}} \leq 3$.

From the above, we see that Theorem 7.1 does not apply. Therefore, let us use Theorems 6.3 and 6.4 to bound the spline space dimension from below and above. Computing the Euler characteristic of $\mathcal{Q}$ to be $\chi(\mathcal{Q})_{m}=143$, we use those theorems to obtain the following:

$$
143 \leq \operatorname{dim}\left(\mathcal{S}_{\Delta m}^{r}\right)_{m} \leq 146
$$


The reader can use the accompanying Macaulay2 script [34, ex3.m2] to confirm that the dimension of the spline space is exactly 146 for this configuration and thus coincides with the computed upper bound. This example serves to show that there exist configurations where a maximal segment ordering that allows us to use Theorem 7.1 does not exist. Indeed, the accompanying script can be used to verify that $\operatorname{dim}\left(H_{0}\left(\mathcal{I}_{[i]}\right)\right)_{m}-\operatorname{dim}\left(H_{0}\left(\mathcal{C}_{[i]}\right)\right)_{m}=3$ for all bi-degrees greater than or equal to $(6,6)$.

\section{Conclusions}

Splines have been used for geometric modeling for several decades, and they are now rapidly becoming indispensable tools for performing approximation. In order to efficiently alter the local resolution offered by a spline space, it is important to be able to perform local adaptivity. While local mesh adaptivity on quadrilateral meshes has been widely studied since the introduction of T-splines [29], theoretical studies focused on splines that allow local degree adaptivity have been missing heretofore from the bivariate spline literature. Since the possibility of using nonuniform bi-degree splines on T-meshes would enable powerful new paradigms of local refinement, we take a first step in this direction by analyzing the dimension of such spline spaces. In particular, using tools from homological algebra, we provide combinatorial bounds on the dimension. We also outline sufficient conditions that guarantee that the spline space dimension is stable, i.e., the dimension of the space is independent of the geometry of the T-mesh for a fixed topology. Several examples are provided to show applicability of the theory developed here.

The results presented in this paper can be used for classifying spline spaces with stable dimension. This is important for avoiding geometry-based linear dependency issues that may arise when performing approximation. The ability to combinatorially compute the spline space dimension is also important because it can be used to determine when a given set of linearly independent splines spans the full spline space. Conversely, given a constructive approach that aims to produce linearly independent splines over T-meshes using only local data, computation of the associated spline space dimension can help identify cases where the splines produced by the approach cannot be linearly independent. This is crucial for devising constructive approaches that can be robustly employed for performing isogeometric analysis.

Current research on this topic is progressing along several lines of inquiry. An interesting direction is the study of local refinement algorithms for both mesh sizes and polynomial degrees that ensure stability of the spline space dimension. The construction of a suitable basis that possesses B-spline-like properties remains an open and essential question and will be a part of future efforts focused on formulation of constructive approaches. A practical construction of this nature has been successfully devised for univariate non-uniform degree splines [30, 36, 37]. A generalization of this univariate approach to the bivariate setting has been recently conjectured [33]. 
Acknowledgments The work of Bernard Mourrain is related to the project GRAPES that has received funding from the European Union's Horizon 2020 research and innovation programme under the Marie Skłodowska-Curie grant agreement No. 860843.

Open Access This article is licensed under a Creative Commons Attribution 4.0 International License, which permits use, sharing, adaptation, distribution and reproduction in any medium or format, as long as you give appropriate credit to the original author(s) and the source, provide a link to the Creative Commons licence, and indicate if changes were made. The images or other third party material in this article are included in the article's Creative Commons licence, unless indicated otherwise in a credit line to the material. If material is not included in the article's Creative Commons licence and your intended use is not permitted by statutory regulation or exceeds the permitted use, you will need to obtain permission directly from the copyright holder. To view a copy of this licence, visit http://creativecommonshorg/licenses/by/4.0/.

\section{References}

1. Bazilevs, Y., Calo, V., Cottrell, J., Evans, J., Hughes, T., Lipton, S., Scott, M., Sederberg, T.: Isogeometric analysis using T-splines. Comput. Methods Appl. Mech. Eng. 199(5-8), 229-263 (2010). https://doi.org/10.1016/j.cma.2009.02.036

2. Billera, L.J.: Homology of smooth splines: generic triangulations and a conjecture of strang. Trans. Amer. Math. Soc. 310(1), 325-340 (1988)

3. Billera, L.J., Rose, L.L.: A dimension series for multivariate splines. 6(2), 107-128. https://doi.org/10.1007/BF02574678 (1991)

4. Bracco, C., Lyche, T., Manni, C., Roman, F., Speleers, H.: On the dimension of Tchebycheffian spline spaces over planar T-meshes. Comput. Aided Geomet. Des. 45, 151-173 (2016). https://doi.org/10.1016/j.cagd.2016.01.002

5. Bracco, C., Lyche, T., Manni, C., Speleers, H.: Tchebycheffian spline spaces over planar T-meshes: Dimension bounds and dimension instabilities. J. Comput. Appl. Math. 349, 265-278 (2019). https://doi.org/10.1016/j.cam.2018.10.026

6. Bressan, A.: Some properties of LR-splines. Comput. Aided Geomet. Des. 30(8), 778-794 (2013). https://doi.org/10.1016/j.cagd.2013.06.004

7. Bressan, A., Buffa, A., Sangalli, G.: Characterization of analysis-suitable T-splines. Comput. Aided Geomet. Des. 39, 17-49 (2015). https://doi.org/10.1016/j.cagd.2015.06.007

8. Buffa, A., Cho, D., Sangalli, G.: Linear independence of the T-spline blending functions associated with some particular T-meshes. Comput. Methods Appl. Mech. Eng. 199(23-24), 1437-1445 (2010). https://doi.org/10.1016/j.cma.2009.12.004

9. Cottrell, J.A., Hughes, T.J.R., Bazilevs, Y. Isogeometric Analysis: Toward Integration of CAD and FEA, 1st edn. Wiley, UK (2009)

10. Deng, J., Chen, F., Li, X., Hu, C., Tong, W., Yang, Z., Feng, Y.: Polynomial splines over hierarchical T-meshes. Graph. Model. 70(4), 76-86 (2008). https://doi.org/10.1016/j.gmod.2008.03.001

11. Dokken, T., Lyche, T., Pettersen, K.: Polynomial splines over locally refined box-partitions. Comput. Aided Geomet. Des. 30, 331-356 (2013)

12. Dokken, T., Lyche, T., Pettersen, K.F.: Polynomial splines over locally refined box-partitions. Comput. Aided Geomet. Des. 30(3), 331-356 (2013). https://doi.org/10.1016/j.cagd.2012.12.005

13. Forsey, D.R., Bartels, R.H.: Hierarchical B-spline refinement. In: ACM SIGGRAPH Computer Graphics, vol. 22, pp. 205-212. ACM (1988). http://dl.acm.org/citation.cfm?id=378512

14. Giannelli, C., Jüttler, B., Speleers, H.: THB-splines: The truncated basis for hierarchical splines. Comput. Aided Geomet. Des. 29(7), 485-498 (2012). https://doi.org/10.1016/j.cagd.2012.03.025

15. Grayson, D.R., Stillman, M.E.: Macaulay2, a software system for research in algebraic geometry. Available at https://faculty.math.illinois.edu/Macaulay2/ (1992)

16. Hatcher, A.: Algebraic Topology. Cambridge University Press, Cambridge (2002)

17. Johannessen, K.A., Kvamsdal, T., Dokken, T.: Isogeometric analysis using LR B-splines. Comput. Methods Appl. Mech. Eng. 269, 471-514 (2014). https://doi.org/10.1016/j.cma.2013.09.014

18. Kraft, R.: Adaptive and linearly independent multilevel B-splines. In: MéhautÉ, A., Rabut, C., Schumaker, L. (eds.) Surface Fitting and Multiresolution Methods, pp. 209-218. Vanderbilt University Press, Vanderbilt (1997) 
19. Li, X., Chen, F.: On the instability in the dimension of splines spaces over t-meshes 28(7), 420-426. https://doi.org/10.1016/j.cagd.2011.08.001. http://www.sciencedirect.com/science/article/ pii/S0167839611000896 (2011)

20. Li, X., Zheng, J., Sederberg, T.W., Hughes, T.J.R., Scott, M.A.: On linear independence of t-spline blending functions 29(1), 63-76. https://doi.org/10.1016/j.cagd.2011.08.005 (2012)

21. Liu, L., Casquero, H., Gomez, H., Zhang, Y.J.: Hybrid-degree weighted T-splines and their application in isogeometric analysis 141, 42-53 (2016). https://doi.org/10.1016/j.compfluid.2016.03.020 (2016)

22. Mourrain, B.: On the dimension of spline spaces on planar t-meshes. Math. Comput. 83(286), 847-871 (2014)

23. Schenck, H.: Computational Algebraic Geometry, vol. 58. Cambridge University Press, Cambridge (2003)

24. Schenck, H., Stillman, M.: Local cohomology of bivariate splines. J. Pure Appl. Algebra 117, 535-548 (1997)

25. Schumaker, L.L.: Bounds on the dimension of spaces of multivariate piecewise polynomials. Rocky Mountain J. Math. 14(1), 251-264 (1984)

26. Schumaker, L.L., Wang, L.: Approximation power of polynomial splines on T-meshes. Comput. Aided Geomet. Des. 29(8), 599-612 (2012). https://doi.org/10.1016/j.cagd.2012.04.003

27. Scott, M., Li, X., Sederberg, T., Hughes, T.: Local refinement of analysis-suitable T-splines. Comput. Methods Appl. Mech. Eng. 213-216, 206-222 (2012). https://doi.org/10.1016/j.cma.2011.11.022

28. Sederberg, T.W., Zheng, J., Bakenov, A., Nasri, A.: T-Splines and T-NURCCs. In: ACM SIGGRAPH 2003 Papers, SIGGRAPH '03, pp. 477-484. ACM, USA (2003). https://doi.org/10.1145/1201775. 882295

29. Sederberg, T.W., Zheng, J., Bakenov, A., Nasri, A.: T-Splines and T-Nurccs. In: ACM Transactions on Graphics (TOG), vol. 22, pp. 477-484. ACM (2003)

30. Speleers, H.: Algorithm 999: Computation of multi-degree b-splines. ACM Trans. Math. Softw. (TOMS) 45(4), 1-15 (2019)

31. Strang, G.: Piecewise polynomials and the finite element method. Bull. Amer. Math. Soc. 79(6), 11281137 (1973)

32. Strang, G.: The dimension of piecewise polynomial spaces, and one-sided approximation. In: Conference on the Numerical Solution of Differential Equations, pp. 144-152. Springer, New York (1974)

33. Thomas, D., Engvall, L., Schmidt, S., Tew, K., Scott, M.: U-splines: Splines over unstructured meshes. Preprint https://coreform.com/usplines (2018)

34. Toshniwal, D.: Supplementary Macaulay2 scripts for splines on T-meshes. Available at https://github. com/dtoshniwal/non-uniform-degree-tsplines-m2 (2019)

35. Toshniwal, D., Hughes, T.J.R.: Polynomial splines of non-uniform degree on triangulations: Combinatorial bounds on the dimension. Comput. Aided Geomet. Des. 75, 101763 (2019)

36. Toshniwal, D., Speleers, H., Hiemstra, R.R., Hughes, T.J.R.: Multi-degree smooth polar splines: a framework for geometric modeling and isogeometric analysis. Comput. Methods Appl. Mech. Eng. 316, 1005-1061 (2017)

37. Toshniwal, D., Speleers, H., Hiemstra, R.R., Manni, C., Hughes, T.J.R.: Multi-degree b-splines: Algorithmic computation and properties. Comput. Aided Geomet. Des. 76, 101792 (2020)

Publisher's note Springer Nature remains neutral with regard to jurisdictional claims in published maps and institutional affiliations. 